\title{
Heterotypic Assembly Mechanism Regulates CHIP E3 Ligase Activity
}

Authors: Aniruddha Das ${ }^{1}$, Pankaj Thapa ${ }^{1}$, Ulises Santiago ${ }^{8}$, Nilesh Shanmugam ${ }^{1}$, Katarzyna Banasiak $^{1}$, Katarzyna Dabrowska ${ }^{3}$, Hendrik Nolte ${ }^{4,6}$, Natalia A. Szulc ${ }^{1}$, Rose M. Gathungu $^{5}$, Dominik Cysewski ${ }^{3}$, Marcus Krüger ${ }^{4,7}$, Michal Dadlez ${ }^{3}$, Marcin Nowotny ${ }^{2}$, Carlos J. Camacho $^{8}$, Thorsten Hoppe ${ }^{4,7}$, Wojciech Pokrzywa ${ }^{1 *}$

\section{${ }^{1}$ Laboratory of Protein Metabolism, International Institute of Molecular and Cell Biology in} Warsaw, Poland

${ }^{2}$ Laboratory of Protein Structure, International Institute of Molecular and Cell Biology in Warsaw, Poland

${ }^{3}$ Institute of Biochemistry and Biophysics, PAS, Warsaw, Poland

${ }^{4}$ Institute for Genetics and Cologne Excellence Cluster on Cellular Stress Responses in AgingAssociated Diseases (CECAD), University of Cologne, Cologne, Germany

${ }^{5}$ Metabolomics Core Facility, EMBL, Heidelberg, Germany

${ }^{6}$ Current: Max-Planck-Institute for Biology of Ageing, Cologne, Germany

${ }^{7}$ Center for Molecular Medicine (CMMC), Faculty of Medicine and University Hospital of Cologne, 50931 Cologne, Germany

${ }^{8}$ Department of Computational and Systems Biology, University of Pittsburgh, Pittsburgh, Pennsylvania

* Correspondence should be directed to:

WP: wpokrzywa@iimcb.gov.pl

\section{ABSTRACT}

The E3 ubiquitin ligases CHIP/CHN-1 and UFD-2 team up to accelerate ubiquitin chain formation. However, it remained largely unclear how the high processivity of this E3 set is achieved. Here we studied the molecular mechanism and function of the CHN-1/UFD-2 complex in Caenorhabditis elegans. Our data show that UFD-2 binding promotes the cooperation between $\mathrm{CHN}-1$ and ubiquitin-conjugating E2 enzymes by stabilizing the CHN-1 U-box dimer. The HSP-1 chaperone outcompetes UFD-2 for CHN-1 binding and promotes the auto-inhibited $\mathrm{CHN}-1$ state by acting on the conserved position of the U-box domain. The interaction with UFD-2 enables CHN-1 to efficiently ubiquitinate S-Adenosylhomocysteinase (AHCY-1), an enzyme crucial for lipid metabolism. Our results define the molecular mechanism underlying the synergistic cooperation of CHN-1 and UFD-2 in substrate ubiquitylation.

\section{KEYWORDS}

C. elegans; ubiquitin; proteostasis; E3; CHIP; UFD-2; HSP70; AHCY; lipids 


\section{HIGHLIGHTS}

- E3 ligase UFD-2 stimulates ubiquitylation activity of CHIP/CHN-1

- UFD-2 binding promotes dimerization of CHIP/CHN-1 U-box domains and utilization of E2 enzymes

- HSP70/HSP-1 by latching the U-box and TPR domains stabilizes the autoinhibitory state of CHIP/CHN-1, limiting interactions with E2s and UFD-2

- Assembly with UFD-2 enables CHIP/CHN-1 to regulate lipid metabolism by ubiquitylation of S-Adenosylhomocysteinase

\section{INTRODUCTION}

The ubiquitin-proteasome system (UPS) includes a well-studied enzymatic cascade that transfers the small protein ubiquitin (Ub) onto a protein substrate (Kerscher et al., 2006). The last step in the UPS enzymatic cascade is mediated by ubiquitin-ligases (E3s), the largest and most diverse group of proteins within the UPS responsible for substrate selection and specificity (Komander, 2009; Buetow \& Huang, 2016). In some instances, other proteins, i.e., ubiquitin chain elongation factors, or E4s, can be required to achieve efficient polyubiquitylation (poly-Ub) of model substrates. The first described E4 was yeast Ufd2p (Richly et al., 2005), a U-box domain-containing protein that engages $\mathrm{Ub}$ via its $\mathrm{N}$-terminal region to assist with $\mathrm{Ub}$ chain elongation on pre-ubiquitylated substrates (Koegl et al., 1999; Hatakeyama et al., 2001; Buetow \& Huang, 2016).

CHIP (C-terminus of Hsc70 interacting protein), initially identified as a tetratricopeptide repeat (TPR) protein that interacts with heat shock proteins (Ballinger et al., 1999), is a U-box E3 ubiquitin ligase that mediates ubiquitylation of chaperone client proteins, resulting in their degradation (Murata et al., 2001). Early Caenorhabditis elegans studies showed that UFD-2 interacts directly with CHN-1 (the nematode homolog of mammalian CHIP) to form an E3/E4 complex that can efficiently oligo-ubiquitylate the myosin chaperone UNC-45 (Hoppe et al., 2004). By contrast to the model proposed based on these early findings, more recent studies have revealed that UFD-2 acts as a true E3 ligase that poly-ubiquitylates UNC-45 independent of $\mathrm{CHN}-1$, suggesting that both UFD-2 and CHN-1 act as E3s in the same or overlapping substrate space (Hellerschmied et al., 2018). A recent study aimed at identifying substrates of human CHIP and the human UFD-2 ortholog UBE4B supports the possibility of shared substrate scope (Bhuripanyo et al., 2018). However, despite the vital role of $\mathrm{CHN}-1 / \mathrm{CHIP}$ in protein quality control networks, little is known about its interactions with E3s and the regulation of its activity. To address these questions, we combined in vitro and in vivo assays with computational approaches and lipidomic and proteomic studies in C. elegans and uncovered the mechanism that controls CHN-1 activity. Our results indicate that UFD-2 interacts with the TPR domain of CHN-1 to boost CHN-1 processivity. This binding stabilizes the open conformation of the CHN-1 dimer enabling the U-box dimer to discharge more Ub-conjugating enzymes (E2) in a single ubiquitylation cycle. We also 
82 demonstrated that the heat shock protein HSP-1 interacts with the TPR and U-box domain of CHN-

831 to stabilize the closed/auto-inhibitory state of CHN-1 dimer and limits its interaction with E2s

84 and UFD-2. Furthermore, we identified potential substrates for the CHN-1/UFD-2 pair, including

$85 S$-adenosylhomocysteinase (AHCY-1), a metabolic enzyme not known to be a client of known heat

86 shock chaperones. Collectively, our results indicate an interplay between chaperones and UFD-2 in modulating CHIP activity. This processivity switching behavior of $\mathrm{CHN}-1$ has important implications for its roles in regulating proteostasis, metabolism, and potentially other cellular processes.

90

\section{RESULTS}

92

\section{UFD-2 promotes CHN-1 processivity and cooperation with E2s}

Binding between CHN-1 and UFD-2 was previously demonstrated via yeast two-hybrid and in vitro pull-down assays (Hoppe et al., 2004). Beyond the physical interaction, the molecular regulation of $\mathrm{Ub}$ chain elongation by the $\mathrm{CHN}-1 / \mathrm{UFD}-2$ complex has not been explored in detail. We performed an in vitro analysis of the activity of both E3s individually and in pairs. First, we chose E2s with which CHN-1 and UFD-2 cooperate in the auto-ubiquitylation (auto-Ub) reaction. Mammalian CHIPs can functionally interact with various E2s, particularly members of the UBCH5/UBE2D family (UBCH5a/UBE2D1, -b/2 and -c/3) (Jiang et al., 2001; Soss et al., 2011). insulin/insulin-like growth factor 1 (IGF-1) receptor in vitro (Tawo et al., 2017). To study the activity of $\mathrm{CHN}-1$ in detail, we compared its ability to self-ubiquitylate in the presence of each of the UBE2D family proteins separately. We observed that $\mathrm{CHN}-1$ cooperated effectively with UBE2D1. However, when we performed an auto-Ub reaction with both E3s, we observed a significant increase in $\mathrm{CHN}-1$ poly-Ub activity, even when the $\mathrm{E} 2$ used in the reaction was UBE2D3, which is not efficiently used by CHN-1 alone (Figure 1A). Furthermore, the presence of UFD-2 also increases CHN-1 activity with the UBE2N/UBE2V1 E2 complex (Fig. S1B), which catalyzes the formation of free Ub chains that are then transferred to substrate proteins (Soss et al., 2011). We also concluded that the induction of E3 ligase activity is unidirectional since we did not detect any significant changes in the auto-Ub of UFD-2 under the same conditions (Fig. S1A). To gain insight into $\mathrm{CHN}$-1processivity, we performed a time-dependent auto-Ub experiment. We observed no notable changes in the amount of ubiquitylated CHN-1 over time (from 60-180 min); however, the presence of UFD-2 strongly increased both mono- and poly-Ub of CHN-1 at the earliest time point (60 min) (Fig. 1B). Using the inactive $\mathrm{CHN}-1^{\mathrm{H} 218 \mathrm{Q}}$ mutant (Tawo et al., 2017), we observed that auto-Ub CHN-1 is not the result of modification by UFD-2 (Fig. S1C). Additionally, by deleting the TPR domain of CHN-1( $\triangle 110 \mathrm{aa})$, we confirmed its involvement in UFD-2 binding (Hoppe et al., 2004), as we could not observe modulation of the activity of this 
122 Next, we wanted to verify whether UFD-2 can regulate the poly-Ub processivity of CHN-1 123 independent of its E3 activity. To examine this mechanism, we used an inactive, recombinant UFD-

1242 mutant with a P951A substitution (Ackermann et al., 2016). We found that UFD-2 ${ }^{\text {P951A inactivity }}$ 125 is due to its inability to bind an E2 enzyme (Fig. S1E). Finally, we performed a CHN-1 126 ubiquitylation reaction in the presence of UFD-2 ${ }^{\mathrm{P} 951 \mathrm{~A}}$. We detected substantial enhancement in 127 both the mono- (using lysine-less $\mathrm{Ub}(\mathrm{UbK} 0)$ ) and poly-Ub activity of CHN-1, regardless of the 128 type of $\mathrm{Ub}$ chain (wild-type $\mathrm{Ub}$ or variant with substitutions of lysines 29, 48, 63 to arginines 129 (UbKTR) (Fig. 1C). To rule out the possibility that UFD-2 ${ }^{\text {P951A }}$ retained activity, we also used a 130 UFD-2 variant (1-910 aa) lacking the entire U-box domain (909-984 aa). We confirmed that this 131 UFD-2 deletion mutant could stimulate $\mathrm{CHN}-1$ activity, indicating that interaction with some motif

134 Budding yeast Ufd2p can operate as a Ub chain elongation factor by interacting directly with Ub through its N-terminal region (Liu et al., 2017). Although higher eukaryotes have an ortholog of yeast Ufd2p, the Ub-interacting motif has little sequence homology (Hänzelmann et al., 2010; Liu et al., 2017) suggesting that the function of UFD-2 as an E4 is not evolutionarily conserved. To investigate whether the increased activity of the CHN-1/UFD-2 complex might stem from the elongation function of UFD-2, we tested whether UFD-2 retained the ability to interact with Ub using surface plasmon resonance (SPR) experiments. By contrast to Ufd2p, full-length UFD-2 did not bind linear Ub chains (Fig. 1D). This suggests that during evolution, UFD-2 lost its ability to elongate $\mathrm{Ub}$ chains directly. Unlike yeast Ufd2p, and perhaps to compensate for Ub binding loss, UFD-2 can induce processivity of its partner CHN-1 (Fig. 1E).

\section{UFD-2 induces structural gain of function in CHN-1}

To gain mechanistic insight into the role of UFD-2 binding to $\mathrm{CHN}-1$, we performed hydrogendeuterium exchange mass spectrometry (HDX-MS) of the dimerization process of both $\mathrm{CHN}-1$ alone and CHN-1 in the presence of UFD-2 (Fig. 2A and S2A). Available crystal structures of CHIP homologs support our HDX-MS analysis both without a chaperone (Nikolay et al., 2004) and with HSP90 (Zhang et al., 2005). In the absence of a TPR binding chaperone, only the dimer domains are revealed by the crystal structure, with no resolution of either the turn in the coil-coil domain or the TPR domain. Of note, the TPR by itself has only been resolved by NMR, whereas in the presence of an HSP substrate it stabilizes into its crystal form (Zhang et al., 2005). Furthermore, mouse CHIP shows that in one of its monomers, bound TPR is further stabilized against the long helix of its coil-coil domain. We have noted that this interaction is much weaker in CHN-1 (Thorsten Hoppe - personal communication), suggesting a more dynamic interaction in 158 worms. Figure 2A and S2A depicts these states, leading to the following interpretation of our HDX159 MS data, which detects at least three dynamical events at $10 \mathrm{~s}$ and $60 \mathrm{~s}$. Namely, (a) the turn in the 160 coil-coil motif (aa 146-152) is stabilized early on upon dimerization of the coil-coil domains; (b) 161 the TPR domain is stabilized upon recognition by UFD-2, leaving the distal helices aa 21-40 and 162 92-112 exposed to solvent. At later times the stable TPR stabilizes against the long helix of the 
163 coil-coil domain; and, (c) the U-box domain (aa 21-40; 92-112) transitions from a weak interaction 164 with its coil-coil domain to a stable dimer at longer time scales. As shown in Fig. 2A, we argue 165 that contrary to CHIP (Ye et al., 2017; Zhang et al., 2005), CHN-1 folds into a symmetric structure 166 as previously we have indicated that $\mathrm{CHN}-1$ has critical residues that should prevent an asymmetric 167 fold (Thorsten Hoppe - personal communication). Thus, while it has been shown that HSP90 negatively regulates CHIP activity (Narayan et al., 2015), presumably by blocking the E2 binding site of one of the protomers (Zhang et al., 2005), our findings of UFD-2 promoting CHN-1 processivity are consistent with a symmetric CHN-1 that upon binding UFD-2 stabilizes the U-box dimer with two E2 sites available for binding.

We conducted a ubiquitylation assay with increasing molar concentrations of UBE2D1 (0.6-6.2 $\mu \mathrm{M})$ to confirm this model. We observed that at a constant Ub concentration, increasing E2 concentration led to an increase in $\mathrm{CHN}-1$ activity. However, even at the highest $\mathrm{E} 2$ concentration $(6.2 \mu \mathrm{M}), \mathrm{CHN}-1$ processivity did not reach the same level as in the presence of inactive UFD$2^{\mathrm{P} 951 \mathrm{~A}}$ and at an approximately 10 -fold lower E2 concentration $(0.6 \mu \mathrm{M})$ (Fig. $\left.2 \mathrm{~B}\right)$. Thus, the increased $\mathrm{CHN}-1$ activity in the $\mathrm{CHN}-1 / \mathrm{UFD}-2$ complex was not due to the increased local E2 concentration but rather to the enhanced processivity of the E2 enzyme bound to the CHN-1 Ubox. To verify this hypothesis, we performed an E2-discharging assay in the presence of CHN-1 alone or after mixing with UFD-2 ${ }^{\mathrm{P} 951 \mathrm{~A}}$ to track the use of charged-E2 by CHN-1 only. We observed that in the presence of UFD-2 ${ }^{\mathrm{P} 951 \mathrm{~A}}, \mathrm{CHN}-1$ could discharge almost twice as much of UBE2D1-Ub (approximately $6.6 \mu \mathrm{M}$ ) compared to $\mathrm{CHN}-1$ alone (approximately $3.9 \mu \mathrm{M}$ ), as indicated by the accumulation of unused UBE2D1-Ub (Fig. 2C). Next, we performed another E2-discharging assay over time (0-30 $\mathrm{min}$ ) to verify whether the increased utilization of charged E2 by the CHN-1/UFD$2^{\mathrm{P} 951 \mathrm{~A}}$ pair was due to altered E2-E3 ubiquitin transfer dynamics. We noted that within the initial 5 minutes, the system achieved the maximum usage of charged E2, and no significant change in the level of available UBE2D1-Ub was observed over time (Fig S2B). Summarizing, our data indicate that UFD-2 acts as a preconditioner for the conformational flexibility of CHN-1, promoting dimerization of the U-box domains and thereby enabling their full functionality.

\section{HSP-1 and UFD-2 modulate CHN-1 processivity by stabilizing its inactive and active conformations, respectively}

The three TPR domains in CHIP act as a binding platform for C-terminal peptides in the Hsp70 and Hsp90 chaperones, containing a conserved EEVD motif (Zhang et al., 2005; Paul \& Ghosh, 2014; Zhang et al., 2015). Since CHN-1 also binds UFD-2 via the TPR domain, we investigated whether HSP-1 (the nematode Hsp70 orthologue) or DAF-21 (the nematode Hsp90 orthologue) could interfere with the activity of the CHN-1/UFD-2 complex. We first examined protein-protein interactions between CHN-1 and UFD-2, HSP-1, or DAF-21 using enzyme-linked immunosorbent assays (ELISAs). CHN-1 showed a higher affinity for HSP-1 and DAF-21 compared to UFD-2 (Fig. S3A). To verify the influence of HSP-1 and DAF-21 on the activity of the CHN-1/UFD-2 
reduced the auto-Ub activity of $\mathrm{CHN}-1$ and blocked the stimulatory capacity of UFD-2 in this process (Fig. 3A). Negative regulation of CHIP auto-Ub by HSP70 has been previously reported, but the molecular basis is unclear (Narayan et al., 2015). Removal of the C-terminal EEVD motif deprived HSP-1 of its inhibitory effect. By contrast, DAF-21 did not affect the UFD-2-enhanced activity of CHN-1 (Fig. 3A).

Next, we performed peptide mapping on peptide microarrays to pinpoint the interaction interface between the two ligases. For this, we used purified CHN-1 tagged with His::SUMO and His::SUMO alone (as control). These proteins were incubated on two UFD-2 peptide microarrays consisting of peptides of lengths 7 and 13 aa. This was followed by staining with secondary and control antibodies and reading using the LI-COR Odyssey Imaging System. Signal enrichment analysis suggested that the two consensus sequences EAKAELEEE and EEYDDVPE, were the predominant interactor motif. HSP70/90 uses a similar acidic C-terminal peptide with an EEVD sequence to bind to the TPR domain of target proteins (Scheufler et al., 2000; Gazda et al., 2013), and HSP-1 C- terminal EEVD peptide affected CHN-1 activity (Fig. 3B). Therefore, we examined whether the identified UFD-2 peptides could also regulate $\mathrm{CHN}-1$. To this end, we performed CHN-1 auto-Ub reactions in the presence of the UFD-2-derived peptides identified in the peptide microarray data. We found that only the KKEYEAKAELEEEYDDVP peptide from UFD-2 significantly stimulated CHN-1 auto-Ub (Fig. 3C). An EEYD sequence is present in this peptide, suggesting that UFD-2 can utilize an EEVD-like motif for CHN-1 binding. Furthermore, multiple sequence alignment analysis revealed that in the EEYD motif of UFD-2, the amino acid Tyr (Y) is evolutionarily conserved among higher eukaryotes (Fig. S3B). To further define the functional role of the EEYD motif from UFD-2, we generated a chimeric recombinant HSP-1 protein carrying Nterminal EEYD instead of EEVD. Strikingly, we observed stimulation of CHN-1 auto-Ub when EEYD was introduced into HSP-1 - an opposite effect compared with that of wild-type HSP-1, which inhibited the reaction (Fig. S3C). Chimeric HSP-1 ${ }^{\text {EEYD }}$ also exerted a slightly stimulatory effect on UBE2D1-Ub discharging by CHN-1 (Fig. S3D). This suggests that the CHN-1 activity switch can be regulated by its binding partners' EEV(Y)D motifs.

To assess the contribution of the particular regions of the CHN-1 TPR domain, we generated its truncation variants. We showed that the first 87 amino acids (aa) $(\Delta 87)$ are not responsible for the interaction with UFD-2 and HSP-1, and therefore are not involved in the modulation of CHN-1 processivity. In contrast, removing the subsequent eight residues ( $\Delta 95$ variant) abrogated the $\mathrm{CHN}$ 1 poly-autoubiquitylation activity. Interestingly, the stimulating effect of UFD-2 was still observed, as evidenced by an increase in monoubiquitylated $\mathrm{CHN}_{-1}{ }^{\Delta 95}$ (Fig. S3E). CHN-1 ${ }^{\Delta 95}$ has residues that may be involved in the interaction with UFD-2, including D110 and subsequent twists and helices (Fig. S3F). Indeed, the CHN-1 ${ }^{\Delta 110}$ mutant, which lacks D110, does not show any gain of activity in the presence of UFD-2 (Fig S1D). It is known that a position homologous to D110 in mouse CHIP (D135) is involved in the binding of HSP's, which suggests that this residue is also important for the interaction with UFD-2 EEYD peptide (Fig. S3F). 
245 To understand why HSP-1 and UFD-2 peptides exhibit distinct effects on CHN-1 activity, we

246 looked closely at the mechanism by which increased HSP90 and HSP70 concentration reduces

247 CHIP activity (Narayan et al., 2015). As noted, HSP90 stabilizes an autoinhibit monomer in mouse

248 CHIP (Zhang et al., 2005). Strikingly, this state entails a salt-bridge bridge between HSP90 D501 and CHIP R273, latching the U-box and TPR domains (Fig. S3G). This observation suggests that chaperone binding can directly restrain U-box from participating in Ub processivity. To show that a similar mechanism is at play in inhibiting ubiquitylation by HSP-1, we mutated R230 (homologous position to R273 in CHIP) to alanine to weaken the CHN-1 U-box interaction with the HSP-1 peptide and thus abrogate its inhibitory effect. Indeed, we observed significantly reduced inhibition of CHN-1R230A/UFD-2 complex by HSP-1 (Fig. 3D). Moreover, the addition of HSP1 blocked the utilization of charged $\mathrm{E} 2$ by the CHN-1/UFD-2P951A complex (Fig. 3E). This agrees with the model indicating that by interacting with the TPR and U-box domain, HSP-1 stabilizes the autoinhibited state of CHN-1, affecting interaction with E2 enzymes (Fig. 3F). On the other hand, UFD-2 can avoid interacting with R230 by, for example, forming a helix that cannot extend toward the U-box, and induces uncorrelated mobility of the TPR domains with respect to the Ubox domains, promoting the steady-state open conformation of CHN-1 (Fig. 3F).

\section{The CHN-1/UFD-2 pair regulates phosphatidylcholine synthesis via AHCY-1}

We next wished to establish the functional consequences of $\mathrm{CHN}-1 / \mathrm{UFD}-2$ cooperation in vivo. Based on our in vitro studies, we hypothesized that in vivo $\mathrm{CHN}-1$, when functioning alone, would display insufficient poly-Ub activity and mainly mono-Ub substrates. Indeed, Tawo and colleagues showed that CHN-1/CHIP mono-Ub the DAF-2 insulin receptor (Tawo et al., 2017). We further assumed that the interaction with UFD-2 would trigger the poly-Ub activity of CHN-1, consequently leading to efficient degradation of its specific substrates. Thus, to understand the role of CHN-1 and UFD-2 in vivo, we decided to identify such substrates. We searched for proteins whose levels increase after deletion of $\mathrm{CHN}-1$ (substrate ubiquitylation by $\mathrm{CHN}-1$ would be affected directly) or UFD-2 (CHN-1 would not be stimulated to efficiently poly-Ub its substrates). To define the consequences of chn-1 and ufd-2 deletion on the C. elegans proteome and to detect proteins that accumulate in the deletion mutants in an unbiased way, we performed label-free mass spectrometry (LC-MS/MS)-based proteomics experiment. We analyzed chn-1(by155), ufd2(tm1380), and chn-1(by155); ufd-2(tm1380) double-mutant worms by single-shot LC-MS/MS gradients in 5 biological replicates. To obtain a view of the global structure of the data, we performed dimensional reduction using principal component analysis (PCA). We noticed that the proteomes of chn-1(by155) and chn-1(by155); ufd-2(tm1380) mutants clustered closer together than did those of chn-1(by155) and ufd-2(tm1380) (Fig. S4A). We hypothesized that potential substrates should accumulate in all mutants; therefore, we filtered the set of significantly altered proteins requiring a two-fold enrichment in all mutants versus the $\mathrm{N} 2$ control strain. We obtained 65 potential substrate candidates and visualized them via hierarchical clustering (Fig. S4B, C and 
them, we identified the AHCY-1 enzyme (Fig. 4A and S4C). AHCY-1 catalyzes the reversible hydrolysis of $S$-adenosylhomocysteine (SAH) to homocysteine and adenosine (Palmer and Abeles, 1976; 1979) (Fig. 4B). Despite the fundamental role of AHCY-1 in metabolism, its regulatory mechanisms are still enigmatic. In a yeast two-hybrid screen using a $C$. elegans cDNA library, we identified AHCY-1 as the prominent interactor of CHN-1 (Fig. S4E). We confirmed the interaction between the two proteins in worms via co-immunoprecipitation (Fig. S4F). Next, we tested whether AHCY-1 is a CHN-1 substrate by performing in vitro ubiquitylation assays with recombinantly expressed proteins. We confirmed that recombinant AHCY -1 is a specific substrate of CHN-1 that UFD-2 does not ubiquitylate (Fig S4H). Furthermore, in the presence of UFD-2, CHN-1 poly-Ub AHCY-1 more effectively, and the level of this modification was reduced by HSP-1 (Fig. 4C, S4G). The cooperation between $\mathrm{CHN}-1$ and UFD-2 is also consistent with the detection of a similar increase in the AHCY-1 level in chn-1(by155), ufd-2(tm1380), and double mutant worms in our proteomic analysis (Fig. 4A). To further validate this observation, we monitored the endogenous level of AHCY-1 via western blotting of total lysates of wild-type worms, chn-1(by155) and ufd2(tm1380) mutant worms, and worms overexpressing chn-1 treated with the proteasome (MG132) and DUB ( $N$-methylmaleimide, NEM) inhibitors. We did not observe any significant changes in the AHCY-1 level, which, according to our other observations, is a stable and abundant protein in C. elegans. However, immunoblotting analysis with anti-AHCY-1 antibodies detected higher molecular weight smeared bands, likely corresponding to polyubiquitinated AHCY-1 species. These AHCY-1 modifications were more abundant when chn-l was over-expressed and were reduced in chn-1(by155) and ufd-2(tm1380) mutant worms compared with the ACHY-1 status in wild-type animals (Fig. 4D). These data suggest that CHN-1 regulates AHCY-1 via an E3 activity triggered by UFD-2.

Elevated homocysteine levels are linked to the deregulation of lipid metabolism and increased fat accumulation, apparent after RNA interference (RNAi) depletion of AHCY-1 in worms (Vrablik et al., 2015; Visram et al., 2018). Using the lipophilic fluorophore RediStain WormDye Lipid Green to stain and quantify the fat content of $C$. elegans, we confirmed that AHCY-1 depletion increases the abundance of lipids in wild-type worms by almost $60 \%$. Overexpression of chn- 1 caused an increase in total lipid content to the similar level detected in ahcy-1 RNAi-treated worms, and this effect was not further enhanced by AHCY-1 depletion (Fig. 4E). Interestingly, mutations in $c h n-1$ and $u f d-2$ cause a reduction in overall lipid levels and uncouple the stimulation of lipid biogenesis induced by ahcy-1 RNAi (Fig. 4E). Synthesis of phosphatidylcholine (PC) from phosphatidylethanolamine (PE) via the de novo phospholipid methylation pathway requires a significant amount of SAM and is particularly sensitive to SAH levels (Tehlivets, 2011). Consistent with our assumption that deletion of either chn-1 or ufd-2 would positively affect AHCY-1 stability, leading to intensification of SAM-dependent methylation and PE to PC conversion, we noted that the ratio of PC to PE increased in chn-1(by155) and ufd-2(tm1380) worms (Fig. 4F). In conclusion, our data suggest a functional role for the CHN-1/UFD-2 complex in AHCY-1dependent lipid metabolism regulation. 


\section{DISCUSSION}

The different conformations achieved by dynamic and flexible motifs are important for the functionality of various E3 ligases (Faull et al., 2019; Kamadurai et al., 2013; Liu and Nussinov, 2011; Narayan et al., 2015). The crystal structure of murine CHIP E3 bound to an HSP-90 decapeptide containing an EEVD motif revealed an asymmetric dimerization in which the two CHIP protomers adopt different conformations. In this "closed" state, only one of the U-box domains in the dimer is accessible for E2 binding, and the other is blocked by the TPR domain (Zhang et al., 2005). In agreement with a computational model of human CHIP (Ye et al., 2017), our homology modeling of the CHN-1 dimer suggested that it can take the form of both a metastable symmetric dimer in which both U-box domains can simultaneously bind E2 ubiquitinconjugating enzymes and asymmetric dimer with low ubiquitylation activity. We showed that the interaction of E3 UFD-2 with the CHN-1 TPR domain reduces its dynamics and thus its blocking of U-box domains. In this steady-state open conformation, $\mathrm{CHN}-1$ achieved high poly-Ub activity due to the full functionality of the U-box dimer. Consistently, in the E2 discharging assay, we observed a two-fold increase in the utilization of charged E2 by the CHN-1/UFD-2 ${ }^{\mathrm{P} 951 \mathrm{~A}}$ complex compared with that of CHN-1 alone. Here, UFD-2 acts as a pre-conditioning factor to influence the conformational flexibility of $\mathrm{CHN}-1$, allowing higher processivity at the initial phase; therefore, we did not observe any further change in our E2 discharging reaction kinetics with increasing time. Using various ubiquitin variants, we showed that not only poly-Ub but also CHN-1 mono-Ub, which is the rate-limiting step of ubiquitylation, is also enhanced upon UFD-2 binding. We also found that UFD-2 activity is unaffected in the complex and that the two ligases are not substrates for each other.

The N-terminal TPR domain of CHIP has been shown to interact specifically with the C-terminal EEVD motif of HSP70 and HSP90 (Zhang et al., 2005; Xu et al., 2006; Graf et al., 2010). We discovered that UFD-2 uses a slightly modified motif - EEYD to engage the CHN-1 TPR domain. Furthermore, we demonstrated that only the presence of a UFD-2 peptide containing the EEYD sequence was sufficient to promote $\mathrm{CHN}-1$ activity. In contrast, the $C$. elegans $\mathrm{HSP} 70$ homolog, HSP-1, negatively regulates $\mathrm{CHN}-1$ and $\mathrm{CHN}-1 / \mathrm{UFD}-2$ complex activity by promoting its autoinhibited (closed) CHN-1 state. GHFDPVTR sequence in the U-box domain is evolutionarily conserved in CHIP from different species, but its role was not previously known. Here we showed that $\mathrm{CHN}-1$ activity is negatively regulated by the interaction between positions associated with the EEVD motif of HSP-1 and the conserved R230 position in the GHFDPVTR sequence. Through direct interactions with the TPR and U-box domain of CHN-1, HSP-1 brings both regions to proximity impairing the U-box dimer. This depends only on the local interaction of the C-terminus of HSP-1 with the U-box, not on the steric hindrance of E2 access to U-box domains by the whole chaperone. In co-crystal with CHIP, HSP90 also forms hydrogen bonds (H-bonds) between T and $\mathrm{S}$ in its C-terminal peptide (TSRMEEVD) and TPR of CHIP (Zhang et al., 2005). The existence of these H-bonds between the HSP-1 peptide (GPTIEEVD) and CHN-1 is not apparent. However, the C-terminal sequence of HSP-1 is rich in glycines that may tailor the binding more efficiently by 
forming H-bonds with the CHN-1 backbone, possibly leading to a very close interaction. Accordingly, the C-terminal HSP70 peptide blocks CHIP activity markedly greater than the HSP90 peptide, which binds to the CHIP TPR domain weaker than the HSP70 peptide (Narayan et al., 2015). We also observed that worm DAF-21/HSP90 has a lower affinity for CHN-1 and does not affect CHN-1 activity, unlike HSP-1. Thus, the mechanism in which the degree of interaction depends on the $\mathrm{C}$-terminal sequence of the chaperones is conserved and correlates with the stabilization of the autoinhibited CHN-1/CHIP dimer. Presumably, high $\mathrm{CHN}-1$ processivity is undesirable for HSPs as it could lead to an imbalance between chaperone-mediated folding/maturation and degradation, inducing the latter. We cannot exclude the influence of posttranslational factors or the cellular environment on the level of regulation of CHN-1 activity by HSP-1 and UFD-2.

Previous research has established that CHIP participates in protein quality control by routing a wide range of chaperone substrates for degradation (Joshi et al., 2016). Our observations suggest an alternative, non-quality control role for the CHN-1/UFD-2 complex. We identified AHCY-1 as a novel substrate of the CHN-1/UFD-2 complex. AHCY-1 is the only eukaryotic enzyme capable of hydrolyzing SAH, which is essential for SAM-dependent methylation (Cantoni, 1975). Recent findings support the importance of CHIP in regulating the methylation status of the cellular proteome by mediating proteasomal turnover of the SAM-dependent methyltransferases PRMT1, PRMT5, and EZH2 (Zhang et al., 2016; Bhuripanyo et al., 2018). However, further studies are necessary to delineate the involvement of the CHN-1/UFD-2 complex in modulating the cellular methylation potential. In summary, our data provide mechanistic insights into the distinct regulation of $\mathrm{CHN}-1 / \mathrm{CHIP}$ activity by HSP70 and UFD-2 and the processivity of non-chaperone CHIP substrates.

\section{LIMITATIONS OF THE STUDY}

Our study elucidates the possible mechanism of action of CHN-1 in the presence of various partner proteins based on the biochemical data and the available structure of CHIP. However, we were unable to provide detailed insights into the structure of CHN-1 in association with UFD-2 or HSPs as we were unable to obtain a co-crystal after various attempts. Furthermore, we could not isolate the essential amino acids of CHN-1 that are required for interaction with UFD-2 and HSP-1. Therefore, we cannot exclude the possibility that multiple CHN-1 motifs may be involved in the interactions. In addition to presenting a novel, non-quality-controlling role for the CHN-1/UFD-2 pair, we cannot comment on the physiological factors that regulate this E3 complex assembly.

\section{LEGENDS}

Figure 1: UFD-2 activates CHN-1. A) Auto-Ub of recombinant CHN-1 and UFD-2 was carried out using the E2s UBE2D1, UBE2D2, and UBE2D3. CHN-1 ubiquitylation was assessed via 
western blotting using CHN-1-specific antibodies. B) Time-dependent (60, 90, 180 min) auto-Ub of CHN-1 was performed as indicated using wild-type ubiquitin $\left(\mathrm{Ub}^{\mathrm{WT}}\right)$ or a lysine-free variant $\left(\mathrm{Ub}^{\mathrm{NoK}}\right)$. Protein samples were resolved via SDS-PAGE and immunoblotted with anti-CHN-1 antibodies. C) Auto-Ub was performed as indicated using recombinant CHN-1 and UFD-2 ${ }^{\text {P951A }}$, UBE2D1 E2, $\mathrm{Ub}^{\mathrm{WT}}, \mathrm{Ub}^{\mathrm{NoK}}$ or $\mathrm{Ub}$ with substitutions of lysines $29,48,63$ to arginines ( $\mathrm{Ub}^{3 \mathrm{KTR}}$ ). Protein samples were resolved via SDS-PAGE and immunoblotted with anti-CHN-1 antibodies. D) Surface plasmon resonance (SPR) sensorgrams of the interaction between linear di-Ub (M1linear from UbiQ) and C. elegans UFD-2 (red) or S. cerevisiae Ufd2p (blue). Y-axis: response unit $(\mathrm{RU})$ value. $\mathrm{X}$-axis: molar concentration of linear di-Ub. E) In vitro auto-ubiquitylation of CHN-1 in the presence of recombinant $C$. elegans UFD-2 and $S$. cerevisiae Ufd-2p, and UBE2D1 E2. Protein samples were resolved via SDS-PAGE and immunoblotted with anti-CHN-1 antibodies. Immunoblots representative of $n=3$ experiments are shown.

Figure 2: UFD-2 stabilizes CHN-1 U-box dimer. A) HDX-MS was used to analyze changes in the structural dynamics of residues within CHN-1 when in complex with UFD-2. The diagram model represents regions of retarded (red) and enhanced (blue) exchange in CHN-1 (upper panel). (colored box: blue, light red, medium red, dark red) in the different domains upon interaction with UFD-2 (lower panel). B) Auto-Ub of CHN-1 was performed as indicated using increasing amounts $(0.6,1.2,2.5,6.2 \mu \mathrm{M})$ of UBE2D1 or $0.6 \mu \mathrm{M}$ UBE2D1 after complexing CHN-1 with $0.2 \mu \mathrm{M}$ of anti-CHN-1 antibodies. Quantification of the change in unmodified CHN-1 levels. Y-axis against the intensity of the unmodified band in each lane. Analysis performed using GraphPad Prism. C) E2 discharging assay of Ub-charged UBE2D1 in the presence of CHN-1/UFD-2 ${ }^{\mathrm{P} 951 \mathrm{~A}}$. The reaction was stopped after the indicated time via heat inactivation in native conditions. Protein samples were resolved via SDS-PAGE and immunoblotted with anti-Ub antibodies. (left panel). signal from each and $\mathrm{X}$-axis plotted against the $\mu \mathrm{M}$ concentration of UBE2D1-Ub (right panel). Immunoblots representative of $n=3$ experiments are shown.

Figure 3: UFD-2 stabilizes an open/active, and HSP-1 stabilizes a closed/non-active CHN-1 conformation. A) In vitro auto-Ub was performed as indicated using recombinant $\mathrm{CHN}-1$ complexed with UFD-2 in the presence of recombinant DAF-21, DAF-21 $\triangle$ EEVD, HSP-1, or HSP$1 \triangle E E V D$. Protein samples were resolved via SDS-PAGE and immunoblotted with anti-CHN-1 antibodies. B) On top schematics of the HSP-1 peptide sequence used in ubiquitylation reaction. Auto-Ub was performed as indicated using recombinant $\mathrm{CHN}-1$ and HSP-1 derived peptide. Protein samples were resolved via SDS-PAGE and immunoblotted with anti-CHN-1 antibodies. Below, quantification of the changes (\%) in (un)modified $\mathrm{CHN}-1$ levels. Immunoblots representative of $n=3$ experiments are shown. C) Schematics of the UFD-2 peptide sequences used in further ubiquitylation reactions (left panel). Auto-Ub was performed as indicated using recombinant CHN-1 and UFD-2 derived peptides. Protein samples were resolved via SDS-PAGE 
and immunoblotted with anti-CHN-1 antibodies (middle panel). Quantification of the changes (\%) in (un)modified CHN-1 levels (right panel). D) Auto-Ub was performed as indicated using recombinant $\mathrm{CHN}-1^{\mathrm{R} 230 \mathrm{~A}}$, UFD-2 and HSP-1. Protein samples were resolved via SDS-PAGE and immunoblotted with anti-CHN-1 antibodies. Immunoblots representative of $n=3$ experiments are shown. E) E2 discharging assay of Ub-charged UBE2D1 in the presence of a ternary mixture of recombinant $\mathrm{CHN}-1 / \mathrm{UFD}-2^{\mathrm{P} 951 \mathrm{~A}} / \mathrm{HSP}-1$. The reaction was stopped after the indicated time via heat inactivation in native conditions. Protein samples were resolved via SDS-PAGE and immunoblotted with anti-Ub antibodies. F) Model of the UFD-2 activation and HSP-1 inhibition of CHN-1. Dimeric CHN-1 with TPR, U-box, and helix-turn-helix (HH) indicated by magenta, gold and cyan color, respectively. UFD-2 and HSP-1 peptides in yellow with indicated amino acid positions in the full-length proteins.

Figure 4: The CHN-1/UFD-2 pair regulates lipid metabolism via AHCY-1. A) Endogenous levels of AHCY-1 in N2 (wild-type), chn-1(by155), ufd-2(tm1380), and chn-1(by155); ufd2(tm1380) mutant worms reported as Z-scores from LC-MS/MS analysis. B) Schematic diagram representing the core function of AHCY. AHCY catalyzes the reversible hydrolysis of SAH (Sadenosylhomocysteine) to HCy (Homocysteine). Accumulation of SAH inhibits PC (Phosphatidylcholines) synthesis from PE (Phosphatidylethanolamines). C) Ubiquitylation of recombinant AHCY-1 was performed as indicated using recombinant CHN-1 and UFD-2, UBE2D1 E2, $\mathrm{Ub}^{\mathrm{WT}}$, or $\mathrm{Ub}^{\mathrm{K} 48}$ and $\mathrm{Ub}^{\mathrm{K} 63}$ only Ub variants. Protein samples were resolved via SDSPAGE and immunoblotted with anti-AHCY-1 antibodies. Bands labeled as unmodified AHCY-1, mono-Ub AHCY-1, di-Ub AHCY-1, poly-Ub AHCY-1 (left panel). Quantification of the changes (\%) in (un)modified AHCY-1 levels (right panel). D) Endogenous levels of AHCY-1 in N2 (wildtype), chn-1(by155), CHN-1::FLAG (OE), and ufd-2(tm1380) young adult worms treated with a proteasome inhibitor (MG132, 10 $\mathrm{M}$ ) or DUB inhibitor (NEM, 100mM). Protein samples were resolved via SDS-PAGE and immunoblotted with anti-AHCY-1 antibodies. Tubulin served as a loading control. Immunoblots representative of $n=3$ experiments are shown. E) Total lipid content in N2 (wild-type), chn-1(by155), ufd-2(tm1380), chn-1(by155), ufd-2(tm1380), and CHN-1::FLAG (OE) young adult worms grown on control and ahcy-1 RNAi feeding plates. Data are means \pm SEM, $\mathrm{p} \leq 0.001(* * *)$. Higher fluorescence intensity indicates increased lipid levels. F) Ratio of phosphatidylcholine (PC) to phosphatidylethanolamine (PE) in N2 (wild-type), chn-1(by155), and ufd-2(tm1380) young adult worms.

Supplementary Figure S1: A) Auto-Ub of UFD-2 was performed as indicated using E2s UBE2N/Uev1a, UBE2D1, or UBE2D3, and $\mathrm{Ub}^{\mathrm{WT}}$ or $\mathrm{Ub}^{\mathrm{NoK}}$. Protein samples were resolved via SDS-PAGE and immunoblotted with anti-UFD-2 antibodies. B) Auto-Ub was performed as indicated using UBE2N/Uev1a and UBE2D1 E2s. Protein samples were resolved via SDS-PAGE and immunoblotted with anti-Ub antibodies. C) Ubiquitylation of recombinant $\mathrm{CHN}-1^{\mathrm{H} 218 \mathrm{Q}}$ mutant was performed as indicated. Protein samples were resolved via SDS-PAGE and immunoblotted with anti-CHN-1 antibodies. D) Auto-Ub of recombinant $\mathrm{CHN}-1^{\Delta 110}$ was performed as indicated 
CHN-1 antibodies. E) Co-immunoprecipitation of ubiquitin-charged GST-UBE2D1 from a mixture of recombinant GST-UBE2D1 and CHN-1, GST-UBE2D1 and UFD-2 ${ }^{\text {P951A }}$, and the ternary mixture of GST-UBE2D1, CHN-1 and UFD-2 ${ }^{\mathrm{P} 951 \mathrm{~A}}$ using Dynabeads conjugated with antiGST antibody. Protein samples were resolved via SDS-PAGE and immunoblotted with anti-GST, anti-UFD-2, and anti-CHN-1 antibodies. F) Auto-Ub was performed as indicated using recombinant UFD-2, UFD-2 ${ }^{\mathrm{P} 951 \mathrm{~A}}$, or UFD-2 ${ }^{\text {AUbox }}$. Bands labeled as unmodified $\mathrm{CHN}-1$, mono-Ub $\mathrm{CHN}-1$, and poly-Ub CHN-1. Below, quantification of the changes (\%) in (un)modified $\mathrm{CHN}-1$ levels. Immunoblots representative of $\mathrm{n}=3$ experiments are shown.

Supplementary Figure S2: A) Differential Woods plots present the difference in fractional deuterium uptake between two biological states - $\mathrm{CHN}-1$ and $\mathrm{CHN}-1$ in the presence of wild-type UFD-2. The $\mathrm{X}$-axis represents the position in sequence for a peptide (the $\mathrm{x}$ value indicates the peptide length). The $\mathrm{Y}$-axis presents the difference in fractional deuterium uptake with the $\mathrm{Y}$-error bar indicating the uncertainty of the measurement from three independent replicates of the experiment. Positive values indicate stabilization of the region upon complex formation. Dotted lines indicate the confidence limit at 95\% calculated using the Houde test (Houde et al., 2011). The upper and lower panels show results after 10 and 60 seconds of H/D exchange, respectively. B) E2 discharging assay of Ub-charged UBE2D1 by the recombinant CHN-1/UFD-2 ${ }^{\mathrm{P} 951 \mathrm{~A}}$. The reaction was stopped after the indicated time via heat inactivation in native conditions. Protein samples were resolved via SDS-PAGE and immunoblotted with anti-Ub antibodies.

Supplementary Figure S3: A) Titration ELISA assay to determine the dissociation constant $\left(\mathrm{K}_{\mathrm{D}}\right)$ between DAF-21, HSP-1, UFD-2, and CHN-1. Y-axis: CHN-1 concentration $(\mu \mathrm{M})$. X-axis: absorbance (OD) at $450 \mathrm{~nm}$. Below, a table showing the $\mathrm{K}_{\mathrm{D}}$ value $(\mathrm{nM})$ of the corresponding protein with recombinant CHN-1. B) Multiple sequence alignment (MSA) of UFD-2 orthologs from different species. Orthologous sequences (from Orthologous Group ID ENOG5038DSP) of selected species were obtained from the eggNOG5 database (Huerta-Cepas et al., 2019) and aligned using the T-Coffee web server with default parameters (Di Tommaso et al., 2011; Notredame et al., 2000). Vertebrates possess two UFD-2 orthologs, which have been independently annotated. MSA was visualized in Jalview Desktop software (Waterhouse et al., 2009) with residues colored according to their physicochemical properties; conserved tyrosine $(\mathrm{Y})$ residues and the EEYD motif in C. elegans are highlighted in white frames. C) Auto-Ub was performed as indicated using recombinant $\mathrm{CHN}-1$ complexed with HSP-1 and HSP-1 ${ }^{\text {EEYD }}$. Protein samples were resolved via SDS-PAGE and immunoblotted with anti-CHN-1 antibodies. Immunoblots representative of $n=3$ experiments are shown. D) E2 discharging assay of Ub-charged UBE2D1 in the presence of CHN1 or $\mathrm{CHN}-1 / \mathrm{HSP}-1{ }^{\mathrm{EEYD}}$. The reaction was stopped after the indicated time via heat inactivation in native conditions. Protein samples were resolved via SDS-PAGE and immunoblotted with anti-Ub antibodies. Immunoblots representative of $\mathrm{n}=3$ experiments are shown. E) In vitro autoubiquitylation was performed as indicated using recombinant $\mathrm{CHN}-1^{\Delta 87}$ or $\mathrm{CHN}-1^{\Delta 95}$ truncation mutants in the presence of UFD-2, DAF-21, DAF-21 $\triangle$ EEVD, HSP-1, and HSP- 
531 Immunoblots representative of $n=3$ experiments are shown. F) Model of the CHN-1 TPR domain 532 docked with UFD-2 EEYD peptide with 1-86 residues of CHN-1 colored in orange and 87-95

533 residues in magenta colors that sequester the EEYD motif away from R230 residue of CHN-1. G)

534 Co-crystal structure of the mice CHIP TPR domain showing interaction with HSP90 EEVD peptide

535 (2C2L) shows R273 (conserved in CHN-1 as R230) in proximity close enough to interact with 536 D501 of HSP90.

537

538 Supplementary Figure S4: A) PCA analysis showing the first and second principal components 539 of the significantly altered proteins (ANOVA FDR < 0.05) performed in the Perseus software. The 540 percentage of explained variance is provided on the axis labels as a percentage. B) Schematic 541 representation of the number of identified proteins in a single-shot analysis of LC-MS/MS 542 gradients in 5 biological replicates that led to the identification of proteins with a significant change 543 in abundance in chn-1(by155), ufd-2(tm1380), and chn-1(by155); ufd-2(tm1380) worms (two-fold 544 enrichment in all mutants versus N2 (wild-type) animals). C) Hierarchical clustering of the Z-Score 545 of proteins whose levels increased in chn-1(by155), ufd-2(tm1380), and chn-1(by155); ufd5462 (tm 1380) mutant worms (two-fold enrichment in all mutants versus N2 (wild-type) animals from 547 LC-MS/MS experiment). D) Gene Ontology biological process terms found to be associated with 548 C. elegans genes upregulated (minimum two-fold enrichment versus N2 (control), with FDR < 5490.05 for ANOVA or pairwise t-test) in all mutants; all proteins detected in LC-MS/MS analysis 550 constituted a reference set. Over-representation analysis was performed using the WebGestalt web 551 server with default parameters (Liao et al., 2019). FDR was controlled to 0.25 using the Benjamini552 Hochberg method for multiple testing. E) Yeast 2-hybrid prey fragment analysis. Schematic 553 representations of the AHCY-1 fragments interacting with $\mathrm{CHN}-1$. The coding sequence for $\mathrm{CHN}-$ 5541 was used as bait to screen a random-primed $C$. elegans mixed-stage cDNA library. The selected 555 interaction domain (SID) is the amino acid sequence shared by all AHCY-1 fragments (prey) 556 interacting with $\mathrm{CHN}-1$. The confidence score of this binding (predicted biological score) is A 557 (highest confidence). F) Co-immunoprecipitation of AHCY-1 and UFD-2 from young adult worms 558 expressing CHN-1::FLAG using beads conjugated with anti-FLAG antibody. Protein samples were 559 resolved via SDS-PAGE and immunoblotted with anti-AHCY-1, anti-FLAG, and anti-UFD-2 560 antibodies. (The red boxes mark the protein band). G) Ubiquitylation of recombinant AHCY-1 was 561 performed as indicated using recombinant CHN-1, UFD-2, DAF-21, HSP-1 in the presence of 562 UBE2D1. Protein samples were resolved via SDS-PAGE and immunoblotted with anti-AHCY-1 563 antibodies. H) Ubiquitylation of recombinant AHCY-1 was performed as indicated using 564 recombinant UFD-2 and UBE2D1 E2. Protein samples were resolved via SDS-PAGE and 565 immunoblotted with anti-AHCY-1 antibodies. Immunoblots representative of $n=3$ experiments 566 are shown. 


\section{STAR $\star M E T H O D S$}

\section{Lead Contact}

571

572 Further information and requests for reagents may be directed to and will be fulfilled by Wojciech

573 Pokrzywa (wpokrzywa@iimcb.gov.pl).

574

\section{Materials Availability}

576

Plasmids generated by the authors will be distributed upon request to other researchers.

\section{Data a Availability}

The mass spectrometry proteomics data was deposited to the ProteomeXchange Consortium via the PRIDE partner repository with the dataset identifier PXD028023 (Perez-Riverol et al., 2019).

\section{Generation of recombinant proteins}

All recombinant proteins were produced using a bacterial expression system. $\mathrm{CHN}-1$ and the $\mathrm{CHN}$ 1 variants were expressed and purified from Rosetta ${ }^{\mathrm{TM}} 2$ (DE3) cells. UFD-2, HSP-1, DAF-21, and their variants were expressed and purified from BL21 Star ${ }^{\mathrm{TM}}$ (DE3) cells. Truncations and point mutations in the protein constructs were introduced using the Q5 Site-Directed Mutagenesis Kit (NEB, Cat\#E0552S). Protein expression was induced using $0.4 \mathrm{mM}$ IPTG at $22{ }^{\circ} \mathrm{C}$ for $16 \mathrm{hr}$. Respective induced cell pellets were harvested via centrifugation at $4000 \mathrm{rpm}$ for $20 \mathrm{~min}$ at $4{ }^{\circ} \mathrm{C}$. Cells were lysed in a lysis buffer (20 mM HEPES pH 8, NaCl 300 mM, 2 mM BME, protease inhibitor, and DNase) by sonication. After sonication, the supernatant and pellet fractions were separated via high-speed centrifugation at $14000 \mathrm{rpm}$ for $1 \mathrm{hr}$ at $22{ }^{\circ} \mathrm{C}$. Tagged proteins were purified from the soluble fraction of the cell lysates using appropriate Ni-NTA or GST Hi-trap columns or chitin beads (NEB, Cat\#E6901S). After removing the affinity tags, affinity-purified protein fractions were subjected to gel filtration chromatography (Hiload 16/600 Superdex S200, GE Healthcare) to obtain more than $95 \%$ pure protein fractions for use in subsequent biophysical and biochemical experiments. For the in vitro ubiquitylation reactions, we first generated a site as per the manufacturer protocol (NEB Cat\#E6901S). The lysis buffer used for purifying this variant contained HEPES 20mM, TritonX 0.1\%, 5\% glycerol, $500 \mathrm{mM} \mathrm{NaCl}, \mathrm{pH}$ 8.0. To generate tagless $\mathrm{CHN}-1$ and His-tagged $\mathrm{CHN}-1$, we affinity-purified the proteins using Ni-NTA columns and then collected the dimeric fraction using size-exclusion chromatography (SEC). Furthermore, SUMO and the His-tag were cleaved using SUMO protease treatment (16 hr) at 4 degrees, and 607 


\section{Peptide microarray for protein-peptide interaction studies}

610 This assay was performed by PEPperPRINT GmbH (https://www.pepperprint.com/). The CHN-1 611 and UFD-2 sequences were elongated with neutral GSGSGSG linkers on the C- and N-termini to 612 avoid truncated peptides. The elongated $\mathrm{CHN}-1$ sequence was translated into 7, 10, and 13 amino 613 acid peptides with peptide-peptide overlaps of 6,9 , and 12 amino acids. The elongated UFD-2 614 sequence was translated into 7 and 13 amino acid peptides with peptide-peptide overlaps of 6 and 61512 amino acids. After peptide synthesis, all peptides were cyclized via a thioether linkage between 616 a C-terminal cysteine and an appropriately modified N-terminus. The resulting conformational 617 CHN-1 and UFD-2 peptide microarrays contained 813 and 1,986 different peptides printed in 618 duplicate (1,626 and 3,972 peptide spots), respectively. The peptide array was framed by additional 619 HA control peptides (YPYDVPDYAG, 68 spots for CHN-1 and 130 spots for UFD-2). Samples: 620 His-tagged SUMO CHN-1, His-tagged SUMO and His-tagged UFD-2 proteins. Washing Buffer: 621 TBS, pH 7.5 with $0.005 \%$ Tween 20; washing for 2 x 10 sec after each incubation step. Blocking 622 Buffer: Rockland blocking buffer MB-070 (30 min before the first assay). Incubation Buffer: TBS, $623 \mathrm{pH} 8$ with 10\% Rockland blocking buffer MB-070, $10 \mathrm{mM}$ HEPES, $150 \mathrm{mM} \mathrm{NaCl}$ and $0.005 \%$ 624 Tween 20. Assay Conditions: Protein concentrations of $10 \mu \mathrm{g} / \mathrm{mL}$ and $100 \mu \mathrm{g} / \mathrm{mL}$ in incubation 625 buffer; incubation for $16 \mathrm{~h}$ at $4{ }^{\circ} \mathrm{C}$ and shaking at $140 \mathrm{rpm}$. Secondary Antibody: Mouse anti-6x626 His Epitope Tag DyLight680 (1.0 $\mu \mathrm{g} / \mathrm{mL}) ; 45 \mathrm{~min}$ staining in incubation buffer at RT. Control 627 Antibody: Mouse monoclonal anti-HA (12CA5) DyLight800 (0.5 $\mu \mathrm{g} / \mathrm{mL}) ; 45$ min staining in 628 incubation buffer at RT. Scanner: LI-COR Odyssey Imaging System; scanning offset $0.65 \mathrm{~mm}$, 629 resolution $21 \mu \mathrm{m}$, scanning intensities of 7/7 (red $=700 \mathrm{~nm} /$ green $=800 \mathrm{~nm})$. Microarray Data: 630 Microarray Data Sumo Protein (PEP20205011547).xlsx, Microarray Data Sumo CHN-1 Protein 631 (PEP20205011547).xlsx, Microarray Data UFD-2 Protein (PEP20205011547).xlsx. Microarray 632 Identifier: 002413_05 (CHN-1 microarray, four array copies for one-by-one assays) $002413 \_07$ \& 633 002413_08 (UFD-2 microarray, two array copies for one-by-one assays).

\section{Ubiquitylation assays}

In vitro assays were performed according to an earlier protocol (Hellerschmied et al., 2018). The reactions were run at $30{ }^{\circ} \mathrm{C}$ for 90 minutes using $60 \mu \mathrm{M}$ Ubiquitin (Boston Biochem, Ub ${ }^{\mathrm{WT}} \mathrm{Cat} \# \mathrm{U}$ 100H; Ub ${ }^{\mathrm{NoK}}$, Cat\#UM-NOK; Ub ${ }^{3 \mathrm{KTR}}$, Cat\#UM-3KTR; Ub K48 only, Cat\#UM-K480; Ub K63 only, Cat\#UM-K630) in the presence of $100 \mathrm{nM}$ E1 (UBE1, Boston Biochem, Cat\#E-304), $0.6 \mu \mathrm{M}$ 643 variants), E3 ligase reaction buffer (Boston Biochem, Cat\#B-71), and Energy Regeneration 644 Solution (Boston Biochem, Cat\#B-10). For performing the in vitro reaction in the presence of both 645 the $\mathrm{CHN}-1$ and UFD-2 or His-tagged UFD-2 ${ }^{\mathrm{P} 951 \mathrm{~A}}$, proteins were first pre-incubated at $16^{\circ} \mathrm{C}$ for 30 $646 \mathrm{~min}$ in the presence of $\mathrm{E} 3$ ligase reaction buffer. After that, the remaining reagents were added for 647 the ubiquitylation reaction and incubated at $30{ }^{\circ} \mathrm{C}$ for the indicated time. For substrate 648 ubiquitylation, $C$. elegans AHCY-1 was added as the substrate along with the other reagents and 
649 mixed with pre-incubated CHN-1 or pre-incubated CHN-1/UFD-2 and incubated at $30{ }^{\circ} \mathrm{C}$ for 90 650 mins. For performing the in vitro reaction in the presence of a chaperone, C. elegans $1 \mu \mathrm{M}$ His651 tagged HSP-1, His-tagged DAF-21, or other variants were pre-incubated with CHN-1 or CHN$6521 / \mathrm{UFD}-2$ at $16{ }^{\circ} \mathrm{C}$ for $30 \mathrm{~min}$ in the presence of $1 \mathrm{x} \mathrm{E} 3$ ligase reaction buffer. After that, the 653 remaining reagents were added for the reaction and incubated at $30{ }^{\circ} \mathrm{C}$ for $90 \mathrm{~min}$. After the 654 reaction, SDS-loading dye (Bio-rad, Cat\#1610747), including $\beta$-mercaptoethanol (Sigma, 655 Cat\#M6250), was added to the entire reaction mix, and the samples were incubated at $95{ }^{\circ} \mathrm{C}$ for 5 656 min. Samples were run in $12 \%$ SDS-PAGE gels and blotted with an antibody against the protein 657 of interest.

\section{E2 discharge assays}

661 E2 discharging experimental protocol designed based on a modified method from Page et al., 2012. 662 Discharging of increasing molar concentration $(0.8,1.6,3.3,5,6.6 \mu \mathrm{M})$ of charged UBE2D1 663 (Boston Biochem, UBE2D1-Ub, Cat\#E2-800) was performed at $30{ }^{\circ} \mathrm{C}$ for 40 min in ubiquitin 664 conjugation reaction buffer (Boston Biochem, Cat\#B-70). Similarly, a time-dependent assay was performed using $3.3 \mu \mathrm{M}$ UBE2D1-Ub at $30{ }^{\circ} \mathrm{C}$ for different time points $(5,10,20,30$ mins) with equimolar concentrations $(1 \mu \mathrm{M})$ of $\mathrm{CHN}-1$, His-tagged UFD-2 ${ }^{\mathrm{P} 951 \mathrm{~A}}$ and His-tagged HSP-1. The reaction was stopped by the addition of SDS-loading dye (Bio-Rad, Cat\#1610747) without any reducing agent and incubation at $30{ }^{\circ} \mathrm{C}$ for $5 \mathrm{~min}$. Samples were run in a 15\% SDS-PAGE gel. For detecting the available UBE2D1-Ub in each condition, western blotting was performed using an anti-ubiquitin antibody. Normalized chemiluminescence intensity was obtained after maximum background subtraction from each lane.

\section{Western blotting and quantification}

Protein samples in SDS-loading dye (reducing/non-reducing) were run in $12 \%$ or $15 \%$ acrylamide gels using running buffer ( $25 \mathrm{mM}$ Tris, $190 \mathrm{mM}$ Glycine, $0.1 \%$ SDS) at 120 volts (constant). The wet transfer was done at a constant $200 \mathrm{~mA}$ for $2 \mathrm{hr}$ at room temperature using transfer buffer (25 $\mathrm{mM}$ Tris, $190 \mathrm{mM}$ Glycine, 10\% methanol, $\mathrm{pH} 8.3$ ). Blots were then blocked with 5\% skimmed milk in TBST (50 mM Tris, $150 \mathrm{mM} \mathrm{NaCl}, 0.1 \%$ Tween 20, $\mathrm{pH} 7.5$ ) for $1 \mathrm{hr}$ at room temperature. Blots were incubated with primary antibody prepared in $5 \%$ skimmed milk in TBST at $4{ }^{\circ} \mathrm{C}$, overnight. The blots were then washed three times with TBST for $10 \mathrm{~min}$ each. Finally, the blots were incubated with secondary antibodies prepared in 5\% skimmed milk in TBST for $1 \mathrm{hr}$ at room temperature. The blots were imaged using a ChemiDoc ${ }^{\mathrm{TM}}$ Imaging System (Bio-Rad). All antibodies used in this study are listed in the resource table. We used Image Lab $^{\mathrm{TM}}$ (version 6.0.0 build 25) software for blot quantification using Image Lab and graph plotted using GraphPad Prism 


\section{Enzyme-linked immunosorbent assay (ELISA)}

691

$6922 \mu \mathrm{g} / \mathrm{mL}$ of UFD-2, His-tagged DAF-21, and His-tagged HSP-1 in coating buffer (100 mM $693 \mathrm{NaHCO}_{3}, 32 \mathrm{mM} \mathrm{Na} 2 \mathrm{CO}_{3}, \mathrm{pH}$ 9.2) were immobilized on Nunc-Immuno plates for ELISA (Thermo 694 Fisher Scientific, Cat\#44-2404) overnight at $4{ }^{\circ} \mathrm{C}$. Blocking was performed with $2 \%$ BSA for 1 $695 \mathrm{hr}$ at $25^{\circ} \mathrm{C}$ followed by washing with TBST (0.1\% Tween 20). After incubation with increasing $696 \mathrm{CHN}-1$ concentrations of for $1 \mathrm{hr}$ at $16{ }^{\circ} \mathrm{C}$, unbound $\mathrm{CHN}-1$ was washed away by subsequent 697 TBST washing steps. Interacting proteins were detected using an antibody against CHN-1 (1:5000 698 dilution, overnight $4{ }^{\circ} \mathrm{C}$ ), followed by TBST washing and the addition of an HRP-conjugated 699 secondary antibody. After the final wash, $100 \mu \mathrm{L}$ of pnPP substrate (Alkaline Phosphatase Yellow700 Sigma, Cat\#P7998) was added in the dark. After $15 \mathrm{~min}$, the reaction was stopped by adding 50 $\mu \mathrm{L}$ of $3 \mathrm{M} \mathrm{NaOH}$ and the absorbance was measured at $450 \mathrm{nM}$.

702

703

\section{Modeling and Molecular Dynamics}

704

705

CHN-1 model was generated by homology modeling using a Swiss model server (Waterhouse et al., 2018) with PDB ID 2F42 and 2C2L as the templates. The primary sequence of peptides used for docking on the CHN-1 dimer model was 628-640 HSP-1 (P09446) and 894-911 UFD-2 (Q09349). The protein and peptide complex structures were subjected to an energy minimization strategy using pmem.cuda (Goetz et al., 2012; Salomon-Ferrer et al., 2013) from AMBER18 (Case et al., 2018). We used tLeap binary (part of AMBER18) for solvating the structures in an octahedral TIP3P water box with a $15 \AA$ distance from the structure surface to the box edges, and closeness parameter of $0.75 \AA$. The system was neutralized and solvated in a solution of $150 \mathrm{mM} \mathrm{NaCl}$. AMBER ff14SB force field was used (Maier et al., 2015) and simulations were carried out by equilibrating the system for $1 \mathrm{~ns}$ (NPT), at $1 \mathrm{~atm}, 300 \mathrm{~K}$, followed by 10ns NPT for non-bonded interaction. The particle mesh Ewald (PME) method was used to treat the long-range electrostatic interactions. Hydrogen bonds were constrained using SHAKE algorithm and integration time-step at 2 fs. (Ryckaert et al., 1977).

\section{Hydrogen deuterium exchange mass spectrometry (HDX-MS)}

Prior to HDX-MS reactions, a complex of CHN-1 (3 mg/ml) and His-tagged UFD-2 (2 mg/ml) was formed by mixing the proteins in a 1:1 molar ratio followed by incubation at $25{ }^{\circ} \mathrm{C}$ temperature for 30 min. HDX-MS of CHN-1 and CHN-1 in complex with UFD-2 were performed at five time points during the incubation with deuterium (10 sec, $1 \mathrm{~min}, 5 \mathrm{~min}, 25 \mathrm{~min}, 2 \mathrm{hrs}$ ) in triplicate. $5 \mu \mathrm{l}$ aliquots of protein were added to $45 \mu \mathrm{l}$ of deuterated buffer $(10 \mathrm{mM}$ HEPES, $150 \mathrm{mM} \mathrm{NaCl}$ in $\left.99.99 \% \mathrm{D}_{2} \mathrm{O} ; \mathrm{pH}=8.0\right)$ at room temperature. The exchange reaction was quenched by moving the exchange aliquots to pre-cooled tubes (on ice) containing $10 \mu \mathrm{l}$ of quenching buffer ( $2 \mathrm{M}$ glycine, $4 \mathrm{M}$ guanidine hydrochloride, $100 \mathrm{mM}$ TCEP in $99.99 \% \mathrm{D}_{2} \mathrm{O}, \mathrm{pH} 2.3$ ). After quenching, samples were frozen immediately in liquid nitrogen and kept at $-80{ }^{\circ} \mathrm{C}$ until mass spectrometry 
nano ACQUITY UPLC system equipped with HDX-MS Manager (Waters). Proteins were digested online on $2.1 \mathrm{~mm}$ x $20 \mathrm{~mm}$ columns with immobilized Nepenthesin-2 (AffiPro), for $1.5 \mathrm{~min}$ at 20 passed directly to the ACQUITY BEH C18 VanGuard pre-column from which they were eluted onto the reversed-phase ACQUITY UPLC BEH C18 column (Waters) using a 6-40\% gradient of acetonitrile in $0.01 \%$ of formic acid at a flow rate of $90 \mu \mathrm{l} / \mathrm{min}$ at $0.5{ }^{\circ} \mathrm{C}$. Samples were measured on the SYNAPTG2 HDX-MS instrument (Waters) in IMS mode. The instrument parameters for MS detection were as follows: ESI - positive mode; capillary voltage $-3 \mathrm{kV}$; sampling cone voltage $-35 \mathrm{~V}$; extraction cone voltage $-3 \mathrm{~V}$; source temperature $-80^{\circ} \mathrm{C}$; desolvation temperature $-175^{\circ} \mathrm{C}$; and desolvation gas flow - $800 \mathrm{l} / \mathrm{h}$. The $\mathrm{CHN}-1$ peptide list was obtained using nondeuterated protein samples, processed as described above for HDX experiments, and measured in MSe mode. Peptides were identified using ProteinLynx Global Server Software (Waters). The HDX-MS experiment was analyzed using DynamX 3.0 (Waters) software. The PLGS peptide list was filtered by minimum intensity criteria -3000 and minimal product per amino acid -0.3 . All MS spectra were inspected manually. Final data analysis was carried out using in-house HaDex software (Puchała et al., 2020). Differential deuterium exchange of residues was mapped to the model of $\mathrm{CHN}-1$ generated using the 2C2L CHIP structure on the Swiss model server (https://swissmodel.expasy.org/).

\section{Surface plasmon resonance (SPR)}

751

SPR-based interaction analysis was carried out at $25^{\circ} \mathrm{C}$ on a Biacore ${ }^{\mathrm{TM}} \mathrm{S} 200$ instrument (GE Healthcare, Sweden). Recombinant purified His-tagged UFD-2 and His-tagged Ufd2p proteins were immobilized on NTA Biacore sensor Chips (Series S) at $20 \mu \mathrm{g} / \mathrm{mL}$. Single-cycle kinetics studies were performed by passing increasing concentrations ) (0, 100, 200, 500, 1000 and 2000 $\mathrm{nM}$ ) of analyte M1 diUb conjugates (UbiQ, Cat\#UbiQ-L01) in SPR buffer (10 mM HEPES, 150 $\mathrm{mM} \mathrm{NaCl}, 0.05 \%$ Tween 20, 0.1\% BSA, $50 \mu \mathrm{M}$ EDTA, $\mathrm{pH}$ 8.0). The runs for both proteins were carried out under identical conditions. All injections were compiled in the same sensorgram with the response unit $(\mathrm{RU})$ on $\mathrm{Y}$-axis versus time $(\mathrm{sec})$ on the $\mathrm{X}$-axis.

\section{Preparation of $C$. elegans lysates and co-immunoprecipitation}

Worms were grown at $20^{\circ} \mathrm{C}$. For protein extraction, worms were collected in $\mathrm{M} 9$ buffer and lysed using a lysis buffer (1M KCl, $1 \mathrm{M}$ Tris-HCL pH 8.2, $1 \mathrm{M} \mathrm{MgCl}_{2}, 0.07 \%$ NP-40, 0.7\% Tween-20, $0.1 \%$ gelatine) with protease inhibitor (Roche, Cat\# 11873580001) and in the presence of DUB inhibitor (Sigma-Aldrich, Cat\#E3876). First, worms in lysis buffer were snap-frozen in liquid nitrogen. Next, the frozen samples were sonicated (40\% amplitude, 5 cycles of $30 \mathrm{~s}$ pulses at $30 \mathrm{~s}$ intervals, Vibra-Cell ${ }^{\mathrm{TM}}$ ) on ice. Samples were centrifuged at $13,000 \mathrm{rpm}$ for $15 \mathrm{~min}$ and the supernatants were collected. For co-immunoprecipitation, anti-DYKDDDDK (FLAG tag) magnetic beads (Pierce ${ }^{\mathrm{TM}}$ Anti-DYKDDDDK Magnetic Agarose, Cat\#A36797) were used. $50 \mu 1$ 
1::FLAG-expressing worms was used as the experimental sample and wild-type (N2) worms were used as a negative control. Worm lysates were incubated with equilibrated magnetic beads at $4{ }^{\circ} \mathrm{C}$ for 1 and $2 \mathrm{hr}$ for UFD-2 and AHCY-1 pull down, respectively. After the desired incubations, the beads were washed three times using washing buffer (PBS with $100 \mathrm{mM} \mathrm{NaCl}$ ). Samples were eluted via the addition of SDS-loading dye (Bio-rad, Cat\#1610747) containing $\beta$-mercaptoethanol (Sigma, Cat\#M6250) and boiling for 5 min.

\section{RNA interference (RNAi)}

RNAi was performed using the standard RNAi feeding method and RNAi clones (Kamath and Ahringer, 2003). For experiments, NGM plates supplemented with $1 \mathrm{mM}$ IPTG and $25 \mu \mathrm{g} / \mu \mathrm{L}$ carbenicillin were seeded with HT115 E. coli expressing double-stranded RNA (dsRNA) against the gene of interest or, as a control, bacteria with the empty vector were used. Worms were placed on freshly prepared RNAi plates as age-synchronized L1 larvae.

\section{Proteomics}

Protein digestion: For proteomic analysis, the following young adult strains were utilized: N2, ufd2(tm1380), chn-1(by155) and ufd-2(tm1380); chn-1(by155). For lysis, 4\% SDS in 100 mM HEPES $\mathrm{pH}=8.5$ was used, and the protein concentrations were determined. $50 \mu \mathrm{g}$ of protein was subjected for tryptic digestion. Proteins were reduced (10 mM TCEP) and alkylated (20 mM CAA) in the dark for 45 min at $45{ }^{\circ} \mathrm{C}$. Samples were subjected to SP3-based digestion (Hughes et al., 2014). Washed SP3 beads (SP3 beads (Sera-Mag(TM) Magnetic Carboxylate Modified Particles (Hydrophobic), and Sera-Mag(TM) Magnetic Carboxylate Modified Particles (Hydrophilic)) were mixed equally, and $3 \mu \mathrm{L}$ of beads were added to each sample. Acetonitrile was added to a final concentration of 50\%, and the samples were washed twice using $70 \%$ ethanol $(200 \mu \mathrm{L})$ on an inhouse-made magnet. After an additional acetonitrile wash $(200 \mu \mathrm{L}), 5 \mu \mathrm{L}$ of digestion solution (10 mM HEPES pH 8.5 containing $0.5 \mu \mathrm{g}$ Trypsin (Sigma) and $0.5 \mu \mathrm{g}$ LysC (Wako)) was added to each sample and incubated overnight at $37{ }^{\circ} \mathrm{C}$. Peptides were cleaned on a magnet using $2 \times 200$ $\mu \mathrm{L}$ acetonitrile washes. Peptides were eluted in $10 \mu \mathrm{L}$ of $5 \%$ DMSO in an ultrasonic bath for 10 min. Formic acid and acetonitrile were added to final concentrations of $2.5 \%$ and $2 \%$, respectively. Samples were frozen until LC-MS/MS analysis. Liquid chromatography and mass spectrometry: LC-MS/MS instrumentation consisted of a nLC 1200 coupled to a nanoelectrospray source to a QExactive HF-x (Thermo Fisher Scientific) mass spectrometer. Peptide separation was performed on an in-house-packed column ( $75 \mu \mathrm{m}$ inner diameter, $360 \mu \mathrm{m}$ outer diameter), and the column temperature was maintained at $50{ }^{\circ} \mathrm{C}$ using a column oven (PRSO-V2). The LC buffer system consisted out of $0.1 \%$ formic acid (A) and $0.1 \%$ formic acid in $80 \%$ acetonitrile (B). Peptides were separated using a 90 min gradient applying a linear gradient for $70 \mathrm{~min}$ from 7 to $29 \% \mathrm{~B}$ and then ramped to $65 \% \mathrm{~B}$ within $10 \mathrm{~min}$, followed by a linear increase to $95 \% \mathrm{~B}$ within $5 \mathrm{~min} .95 \% \mathrm{~B}$ was 812 operated in a data-dependent acquisition mode targeting the top 22 peaks for collision-induced 
813 fragmentation and MS2 spectra acquisition. MS1 spectra were acquired in a scan range from 350

814 to $1650 \mathrm{~m} / \mathrm{z}$ allowing a maximum injection time of $20 \mathrm{~ms}$ for an AGC target of 3e6. Spectra were

815 acquired at a resolution of 60,000 (at $200 \mathrm{~m} / \mathrm{z}$ ). Ions were isolated in an isolation window of 1.3

$816 \mathrm{~m} / \mathrm{z}$ using an AGC target of $1 \mathrm{e} 6$ and a maximum injection time of $22 \mathrm{~ms}$. Spectra were acquired at

817 a resolution of 15,000 . The scan range for the MS2 spectra was set to $200-2000 \mathrm{~m} / \mathrm{z}$. The

818 normalized collision energy was 28. Dynamic exclusion was set to $20 \mathrm{~s}$. Data analysis: Acquired

819 raw files were correlated to the Uniprot reference C. elegans proteome (downloaded: 06.2018)

820 using MaxQuant (1.5.3.8) (Cox and Mann, 2008) and the implemented Andromeda search engine

821 (Cox et al., 2011). Label-free quantification and matching between runs were enabled using default

822 settings. Carbamidomethylation of cysteine residues was set as a fixed modification. Oxidation of

823 methionine residues and acetylation of protein N-termini were defined as variable modifications.

824 FDR was controlled using the implemented revert algorithm to $1 \%$ at the protein and the peptide-

825 spectrum match (PSM). To identify significantly changed proteins, we performed a one-way

826 analysis of variance (ANOVA) correcting for multiple testing using a permutation-based approach

827 (FDR < 0.05, \# permutations: 500).

828

829

830

831 The following young adult strains were utilized for lipidomic analysis: N2 (wild-type), ufd-

832 2(tm1380), chn-1(by155). Lipid extraction: Lipids from a homogenized sample comprising 15000

833 worms were extracted using the Folch method as follows: $200 \mu \mathrm{L}$ of methanol was added to each

834 sample followed by $10 \mathrm{~s}$ of vortexing. Next, $500 \mu \mathrm{L}$ of chloroform was added, followed by $10 \mathrm{~s}$

835 vortexing. This was followed by the addition of $200 \mu \mathrm{L}$ of water to each sample to induce phase

836 separation, following by vortexing for $20 \mathrm{~s}$. The samples were then kept in the cold for $10 \mathrm{~min}$. The

837 samples were then centrifuged at $14.500 \mathrm{rpm}$ for $10 \mathrm{~min}$. The bottom layer was then pipetted out,

838 and the solvent was dried under a stream of nitrogen. Prior to LC-MS analysis, the lipid extract

839 was reconstituted in $200 \mu \mathrm{L}$ of 1:1 isopropanol:methanol solution. LC-MS analysis: LC-MS

840 analysis was performed as previously described (Nature Methods volume 14, pages 57-60 (2017)).

841 Briefly, lipid extracts were separated on a Kinetex C18 2.1 x 100 mm, $2.6 \mu \mathrm{m}$ column

842 (Phenomonex, Aschaffenburg, De). Separation was achieved via gradient elution in a binary

843 solvent, Vanquish UHPLC (Thermo Scientific, Bremen, DE). Mobile Phase A consisted of

844 ACN: $\mathrm{H}_{2} \mathrm{O}$ (60:40), while mobile phase B consisted of IPA:ACN (90:10). For positive ionization,

845 the mobile phases were modified with $10 \mathrm{mM}$ ammonium formate and $0.1 \%$ formic acid, while for

846 the negative ionization mode, the mobile phases were modified with $5 \mathrm{mM}$ ammonium acetate and

$8470.1 \%$ acetic acid. A flow rate of $260 \mu \mathrm{L} / \mathrm{min}$ was used for separation, and the column and sample

848 tray were held constant at $30^{\circ} \mathrm{C}$ and $4{ }^{\circ} \mathrm{C}$, respectively. $2 \mu \mathrm{L}$ of each sample was injected onto the

849 LC column. MS Instrumentation: MS analysis was performed on a Q-Exactive Plus Mass

850 Spectrometer (Thermo Scientific, Bremen, DE) equipped with a heated electrospray ionization

851 probe. In both the positive and negative ionization modes, the S-Lens RF level was set to 65, and

852 the capillary temperature was set to $320{ }^{\circ} \mathrm{C}$, and the sheath gas flow was set to 30 units and the

853 auxiliary gas was set to 5 units. The spray voltage was set to $3.5 \mathrm{kV}$ in the negative ionization mode 
and $4.5 \mathrm{kV}$ in the positive ionization mode. In both modes, full scan mass spectra (scan range $\mathrm{m} / \mathrm{z}$ $100-1500, R=35 \mathrm{~K}$ ) were acquired along with data-dependent (DDA) MS/MS spectra of the five most abundant ions. DDA MS/MS spectra were acquired using normalized collision energies of 30,40 , and 50 units $(R=17.5 \mathrm{~K}$ and an isolation width $=1 \mathrm{~m} / \mathrm{z})$. The instrument was controlled Linear Dynamics, A Waters Company, Newcastle upon Tyne, UK) was used for peak picking and chromatographic alignment of all samples, with a pooled sample used as a reference. Lipids were annotated using the Progenesis Metascope Basic Lipids the LipidBlast databases with consideration made only of compounds that had MS/MS data. In both databases, the precursor ion tolerance was set to $10 \mathrm{ppm}$, and the fragmentation ion tolerance was set to $15 \mathrm{ppm}$. Putative lipid identifications were based on manual curation of database matches with fragmentation scores $>10 \%$.

\section{Fluorescent labeling of lipids in C. elegans}

Lipid content in young adult worms was determined by RediStain ${ }^{\mathrm{TM}}$ WormDye Lipid Green (NemaMetrix, Cat\#DYE9439) staining, according to the manufacturer's protocol with incubation for 30 mins at room temperature with shaking. Working dye concentration: $1 \mu 1$ of dye/200 $\mu 1$ of M9 buffer. Worms were protected from light, and several washes in M9 buffer were performed after staining. Immediately after that, imaging was performed on a Nikon SMZ25 microscope after immobilizing worms with tetramizole. Data analysis: Image processing was performed with ImageJ (Fiji) using Binary Mask and Particle Analysis Procedure with background signal subtraction. The graphs were plotted using GraphPad Prism 9.

\section{Yeast two-hybrid screening}

Yeast two-hybrid screening was performed by Hybrigenics Services (http://www.hybrigenicsservices.com). The coding sequence for C. elegans CHN-1 (NM_059380.5, aa 1-266) was PCRamplified and cloned into pB27 as a C-terminal fusion to LexA (LexA-CHN-1). The construct was checked by sequencing the entire insert and used as a bait to screen a random-primed $C$. elegans mixed-stage cDNA library constructed into pP6. pB27 and pP6 were derived from the original pBTM116 (Vojtek and Hollenberg, 1995) and pGADGH (Bartel et al., 1993) plasmids, respectively. 61 million clones (6-fold the complexity of the library) were screened using a mating approach with YHGX13 (Y187 ade2-101::loxP-kanMX-loxP, mat $\square$ ) and L40 $\square$ Gal4 (mata) yeast strains as previously described (Fromont-Racine et al., 1997). 202 His+ colonies were selected on a medium lacking tryptophan, leucine, and histidine and supplemented with $50 \mathrm{mM} \mathrm{3-}$ aminotriazole to prevent bait autoactivation. The prey fragments of the positive clones were amplified by PCR and sequenced at their 5' and 3' junctions. The resulting sequences were used to identify the corresponding interacting proteins in the GenBank database (NCBI) using a fully automated procedure. A confidence score (PBS, for Predicted Biological Score) was attributed to each interaction as previously described (Formstecher et al., 2005). The PBS relies on two different 
895 levels of analysis. First, a local score considers the redundancy and independence of prey fragments 896 and the distribution of reading frames and stop codons in overlapping fragments. Second, a global 897 score considers the interactions found in all of the screens performed by Hybrigenics using the 898 same library. This global score represents the probability of interaction being nonspecific. The 899 scores were divided into four categories for practical use, from A (highest confidence) to D (lowest 900 confidence). A fifth category (E) flags explicit interactions involving highly connected prey 901 domains previously found several times in screens performed on libraries derived from the same 902 organism. Finally, several of these highly connected domains were confirmed as false positives 903 and were tagged as F. PBS scores have been shown to positively correlate with the biological 904 significance of interactions (Rain et al., 2001; Wojcik et al., 2002).

905

\section{Acknowledgments}

907

We thank the Caenorhabditis Genetics Center (funded by the NIH National Center for Research Resources, P40 OD010440) for strains, Addgene for plasmids and Katarzyna Prokop and Marta Niklewicz for technical assistance. We thank Vishnu Balaji and Gabriele Stellbrink of the Hoppe laboratory for discussions and technical support and members of Pokrzywa laboratory for discussions and comments on the manuscript.

\section{Funding}

Work in the W.P. laboratory was funded by the Foundation for Polish Science co-financed by the European Union under the European Regional Development Fund (grant POIR.04.04.00-005EAB/18-00) and additionally supported by Polish National Science Center (grant UMO2016/23/B/NZ3/00753). The equipment used for HDX-MS was sponsored by the National Multidisciplinary Laboratory of Functional Nanomaterials (POIGT.02.02.00-00-025/09-00). Work Research Foundation) under Germany's Excellence Strategy - EXC 2030 - 390661388, FKZ: ZUK81/1 and by the European Research Council (ERC-CoG-616499) to T.H. Diese Arbeit wurde von der Deutschen Forschungsgemeinschaft (DFG) im Rahmen der deutschen Exzellenzstrategie - EXC 2030 - 390661388, FKZ: ZUK81 / 1 und vom Europäischen Forschungsrat (ERC-CoG616499) gefördert. Work in M.K. laboratory was supported by the German Research Foundation (DFG) as part of the Excellence Strategy EXC 2030-390661388. C.J.C. and U.S. were funded by National Institute of Health grants R01-GM097082.

929

\section{Author contributions}

931

932 The project was initiated in the laboratory of T.H. A.D., P.T., N.S., K.B., and W.P. designed and 933 conducted experiments. U.S. and C.J.C. performed structural modeling and simulation analysis.

934 R.M.G. performed the lipidomic analysis. N.A.S performed the bioinformatic analyses. H.N. and 935 M.K. performed the proteomic analyses. K.D, D.C, M.D. performed the HDX-MS studies. W.P. 
936 (with input from M.N.) conceived the project and supervised the study. W.P. (with input from

937 M.N.) secured the funding. W.P. and A.D. wrote the manuscript with input from M.N, T.H and

938 C.J.C. The authors declare no competing financial interests.

939

940

TABLE S1. List of constructs and oligonucleotides used to generate them

\begin{tabular}{|c|c|}
\hline Construct & Primer Sequence $\left(5^{\prime}-3^{\prime}\right)$ \\
\hline \multirow[t]{2}{*}{$\begin{array}{l}\text { pTYB21-MBP::Intein- } \\
\text { UFD-2 }\end{array}$} & $\begin{array}{l}\text { Forward: } \\
\text { GGTGGTTGCTCTTCCAACATGATTGAAGACGAGAAAGCAGG }\end{array}$ \\
\hline & Reverse: GGTGGTCTGCAGTCATTATTTCTTTGAATTTCTTT \\
\hline \multirow{2}{*}{$\begin{array}{l}\text { pET-6xHis::SUMO- } \\
\text { CHN-1 }{ }^{\Delta 110}\end{array}$} & Forward: ATTGAGAACGCCCTCAAAC \\
\hline & Reverse: GCTAGCTAGACCACCAATC \\
\hline \multirow{2}{*}{$\begin{array}{l}\text { pET-6xHis::SUMO- } \\
\text { CHN-1 }{ }^{\triangle 87}\end{array}$} & Forward: TACAGTGAAGCAATAAGCTG \\
\hline & Reverse: GCTAGCTAGACCACCAATC \\
\hline \multirow[t]{2}{*}{$\begin{array}{l}\text { pET-6xHis::SUMO- } \\
\text { CHN-1 }{ }^{\Delta 95}\end{array}$} & Forward: TCCAAAGCGCTCTACCAT \\
\hline & Reverse: GCTAGCTAGACCACCAATC \\
\hline \multirow{2}{*}{$\begin{array}{l}\text { pET-21a-VSV-HSP- } \\
1^{\text {EEYD::6xHis }}\end{array}$} & Forward: ATCGAGGAGTACGACGCGGCC \\
\hline & Reverse: GGCCGCGTCGTACTCCTCGAT \\
\hline \multirow{2}{*}{$\begin{array}{l}\text { pET-21a-VSV-HSP- } \\
1 \Delta \text { EEVD::6xHis }\end{array}$} & Forward: GCGGCCGCACTCGAG \\
\hline & Reverse: TCCTCCGGCGGCTCCTCC \\
\hline \multirow{2}{*}{$\begin{array}{l}\text { pET-21a-6xHis::DAF- } \\
21 \Delta \text { EEVD }\end{array}$} & Forward: TAATGAGGATCCGAATTCGAG \\
\hline & Reverse: CTCAGCTCCCTCAATCTT \\
\hline \multirow{2}{*}{$\begin{array}{l}\text { pET-6xHis::SUMO- } \\
\text { CHN-1 }\end{array}$} & Forward: TCCAGTCACAGCAAAACCACTTAC \\
\hline & Reverse: TCGAAATGGCCAATTCTTC \\
\hline
\end{tabular}


KEY RESOURCES TABLE

\begin{tabular}{|c|c|c|}
\hline REAGENT or RESOURCE & SOURCE & IDENTIFIER \\
\hline \multicolumn{3}{|c|}{ Chemicals, Peptides, and Recombinant Proteins } \\
\hline UBE1 & Boston Biochem & Cat\#E-304 \\
\hline UBE2D1 & Boston Biochem & Cat\#E2-616 \\
\hline UBE2D2 & Boston Biochem & Cat\#E2-622 \\
\hline UBE2D3 & Boston Biochem & Cat\#E2-627 \\
\hline UBE2N/Uev1a & Boston Biochem & Cat\#E2-664 \\
\hline 10X E3 Ligase Reaction Buffer & Boston Biochem & Cat\#B-71 \\
\hline 10X Ubiquitin conjugation Reaction Buffer & Boston Biochem & Cat\#B-70 \\
\hline 10X Energy Regeneration Solution & Boston Biochem & Cat\#B-10 \\
\hline Ubiquitin & Boston Biochem & Cat\#U-100H \\
\hline UbNoK & Boston Biochem & Cat\#UM-NOK \\
\hline Ub3KTR & Boston Biochem & Cat\#UM-3KTR \\
\hline UbK48only & Boston Biochem & Cat\#UM-K480 \\
\hline UbK63only & Boston Biochem & Cat\#UM-K630 \\
\hline M1linked- linear ubiquitin & UbiQ & Cat\#UbiQ-L01 \\
\hline UBE2D1 ubiquitin charged & Boston Biochem & Cat\#E2-800 \\
\hline N-Ethylmaleimide & Sigma-Aldrich & Cat\#E3876 \\
\hline $\begin{array}{l}\text { cOmplete }{ }^{\mathrm{TM}} \text {, EDTA-free Protease Inhibitor } \\
\text { Cocktail }\end{array}$ & Roche & Cat\# 11873580001 \\
\hline MG-132 & Selleckchem & Cat\#S2619 \\
\hline $\begin{array}{l}\text { Alkaline Phosphatase Yellow (pNPP) } \\
\text { Liquid Substrate }\end{array}$ & Sigma-Aldrich & Cat\#P7998 \\
\hline Q5 Site-Directed Mutagenesis Kit & NEB & Cat\#E0552S \\
\hline 4x Laemmli Sample Buffer & Bio-Rad & Cat\#1610747 \\
\hline$\beta$-Mercaptoethanol & Sigma-Aldrich & Cat\#M6250 \\
\hline Dynabeads ${ }^{\mathrm{TM}}$ Co-Immunoprecipitation Kit & Invitrogen & Cat\#14321D \\
\hline $\begin{array}{l}\text { Pierce }{ }^{\mathrm{TM}} \text { Anti-DYKDDDDK Magnetic } \\
\text { Agarose }\end{array}$ & Invitrogen & Cat\#A36797 \\
\hline RediStain ${ }^{\mathrm{TM}}$ WormDye Lipid Green & NemaMetrix & Cat\#DYE9439 \\
\hline IMPACT $^{\mathrm{TM}}$ Kit & NEB & Cat\#E6901S \\
\hline AHCY-1::6xHis & This study & N/A \\
\hline CHN-1 & This study & N/A \\
\hline 6xHis::SUMO::CHN-1 & This study & N/A \\
\hline CHN-1 ${ }^{\Delta 110}$ & This study & N/A \\
\hline $\mathrm{CHN}-1^{\Delta 87}$ & This study & N/A \\
\hline $\mathrm{CHN}-1^{\Delta 95}$ & This study & N/A \\
\hline $\mathrm{CHN}-1^{\mathrm{R} 230 \mathrm{~A}}$ & This study & N/A \\
\hline UFD-2 & This study & N/A \\
\hline 6xHis::UFD-2 & This study & N/A \\
\hline 6xHis::UFD-2 ${ }^{\text {P951A }}$ & (Ackermann et al., 2016) & N/A \\
\hline 6xHis::UFD-2 ${ }^{\triangle \mathrm{Ubox}}$ & This study & N/A \\
\hline
\end{tabular}




\begin{tabular}{|c|c|c|}
\hline 6xHis::Ufd2p & This study & $\mathrm{N} / \mathrm{A}$ \\
\hline HSP-1::6xHis & This study & N/A \\
\hline 6xHis::DAF-21 & This study & N/A \\
\hline HSP-1 $1 \triangle$ EEVD::6xHis & This study & N/A \\
\hline 6xHis::DAF-21 $\triangle \mathrm{EEVD}$ & This study & N/A \\
\hline HSP-1 ${ }^{\mathrm{EEYD}}:: 6 \mathrm{xHis}$ & This study & N/A \\
\hline \multicolumn{3}{|l|}{ Antibodies } \\
\hline Anti-CHN-1 antibody & (Tawo et al., 2017) & N/A \\
\hline Anti-UFD-2 antibody & (Ackermann et al., 2016) & N/A \\
\hline Anti-AHCY-1 antibody & This study & N/A \\
\hline Anti-Ubiquitin antibody & Cell signaling Technology & Cat\#3936s \\
\hline Anti-Histidine antibody & $\begin{array}{l}\text { Santa Cruz Biotechnology, } \\
\text { Inc. }\end{array}$ & Cat\#SC-53073 \\
\hline Anti-GST antibody & Sigma-Aldrich & Cat\#G1160 \\
\hline \multicolumn{3}{|l|}{ Bacterial Strains } \\
\hline E. coli RNAi feeding strain & $\begin{array}{l}\text { Caenorhabditis Genetics } \\
\text { Center }\end{array}$ & HT115(DE3) \\
\hline E. coli feeding strain & $\begin{array}{l}\text { Caenorhabditis Genetics } \\
\text { Center }\end{array}$ & OP50 \\
\hline Ahringer RNAi library & Source BioScience & $\begin{array}{l}\text { C. elegans RNAi } \\
\text { Collection } \\
\text { (Ahringer) }\end{array}$ \\
\hline Rosetta $^{\mathrm{TM}} 2$ (DE3) & Novagen & Cat\#71400 \\
\hline BL21 Star ${ }^{\mathrm{TM}}$ (DE3) & Thermo Fisher Scientific & Cat\#C601003 \\
\hline Top10 & Thermo Fisher Scientific & Cat\#C4040 \\
\hline \multicolumn{3}{|l|}{ Oligonucleotides } \\
\hline $\begin{array}{l}\text { For the list of oligonucleotides, see Table } \\
\text { S1 }\end{array}$ & N/A & N/A \\
\hline \multicolumn{3}{|l|}{ Plasmid construct } \\
\hline pET28a-6xHis::Ufd2p & (Liu et al., 2017) & N/A \\
\hline pET-6xHis::SUMO::CHN-1 & This study & N/A \\
\hline pET28a-6xHis::UFD-2 & This study & N/A \\
\hline pLATE31-AHCY-1::6xHis & This study & $\mathrm{N} / \mathrm{A}$ \\
\hline pET21a-VSV::HSP-1::6xHis & This study & N/A \\
\hline pET21a-6xHis::DAF-21 & This study & N/A \\
\hline pTYB21-MBP::Intein-UFD-2 & This study & N/A \\
\hline pET-6xHis::SUMO-CHN-1 ${ }^{\Delta 110}$ & This study & N/A \\
\hline pET-6xHis::SUMO-CHN-1 ${ }^{\Delta 87}$ & This study & N/A \\
\hline
\end{tabular}




\begin{tabular}{|c|c|c|}
\hline pET-6xHis::SUMO-CHN-1 ${ }^{\Delta 95}$ & This study & N/A \\
\hline pET-21a-VSV-HSP-1 ${ }^{\text {EEYD::6xHis }}$ & This study & N/A \\
\hline pET-21a-VSV-HSP-1 $\Delta E E V D:: 6 x H i s$ & This study & N/A \\
\hline pET-21a-6xHis::DAF-21 $\triangle E E V D$ & This study & N/A \\
\hline pET-6xHis::SUMO-CHN-1 ${ }^{\mathrm{R} 230 \mathrm{~A}}$ & This study & N/A \\
\hline \multicolumn{3}{|l|}{ Experimental Models: Organisms/Strains } \\
\hline C. elegans: Bristol (N2) strain as wild-type & CGC & N/A \\
\hline C. elegans: chn-1(by155)I & CGC & $\begin{array}{l}\text { WormBase ID: } \\
\text { WBVar00000641 }\end{array}$ \\
\hline $\begin{array}{l}\text { C. elegans: unc-119(ed4)III; hhIs136[unc- } \\
119(+) ; \text { chn-1p::chn- } 1:: \text { FLAG] }\end{array}$ & (Tawo et al., 2017) & N/A \\
\hline C. elegans: ufd-2(tm1380)II & CGC & $\begin{array}{l}\text { WormBase ID: } \\
\text { WBVar00250374 }\end{array}$ \\
\hline $\begin{array}{l}\text { C. elegans: chn-1(by155)I; ufd-2(tm1380) } \\
\text { II }\end{array}$ & This study & N/A \\
\hline \multicolumn{3}{|l|}{ Software and Algorithms } \\
\hline Graph Pad Prism & Graph Pad Software, Inc. & www.graphpad.com \\
\hline Image $\mathrm{Lab}^{\mathrm{TM}}$ Version 6.0 .0 build 25 & Bio-Rad Laboratories, Inc. & $\begin{array}{l}\text { www.bio- } \\
\text { rad.com/de- } \\
\text { de/product/image- } \\
\text { lab- } \\
\text { software?ID=KRE6 } \\
\text { P5E8Z }\end{array}$ \\
\hline ImageJ $1.53 \mathrm{c}$ & $\begin{array}{l}\text { Wayne Rasband, NIH, } \\
\text { USA }\end{array}$ & $\begin{array}{l}\text { www.imagej.nih.gov } \\
\text { /ij }\end{array}$ \\
\hline \multicolumn{3}{|l|}{ Materials } \\
\hline Nunc MaxiSorp ${ }^{\mathrm{TM}}$ flat-bottom & Thermo Fisher Scientific & Cat\#44-2404 \\
\hline Hiload 16/600 Superdex S200 & GE Healthcare & Cat\#GE28-9893-35 \\
\hline
\end{tabular}

\section{REFERENCES}

Ackermann, L., Schell, M., Pokrzywa, W., Kevei, É., Gartner, A., Schumacher, B., and Hoppe, T. (2016). E4 ligase-specific ubiquitination hubs coordinate DNA double-strand-break repair and apoptosis. Nat. Struct. Mol. Biol.

Ballinger, C.A., Connell, P., Wu, Y., Hu, Z., Thompson, L.J., Yin, L.-Y., and Patterson, C. (1999). Identification of CHIP, a Novel Tetratricopeptide Repeat-Containing Protein That Interacts with Heat Shock Proteins and Negatively Regulates Chaperone Functions. Mol. Cell. Biol.

Bartel, P., Chien, C.T., Sternglanz, R., and Fields, S. (1993). Elimination of false positives that arise in using the two-hybrid system. Biotechniques.

Bhuripanyo, K., Wang, Y., Liu, X., Zhou, L., Liu, R., Duong, D., Zhao, B., Bi, Y., Zhou, H., 
Chen, G., et al. (2018). Identifying the substrate proteins of U-box E3s E4B and CHIP by orthogonal ubiquitin transfer. Sci. Adv.

961 Buetow, L., and Huang, D.T. (2016). Structural insights into the catalysis and regulation of E3 ubiquitin ligases. Nat. Rev. Mol. Cell Biol. Cantoni, G.L. (1975). Biological methylation: selected aspects. Annu. Rev. Biochem. p.p.b.-range mass accuracies and proteome-wide protein quantification. Nat. Biotechnol. Cox, J., Neuhauser, N., Michalski, A., Scheltema, R.A., Olsen, J. V., and Mann, M. (2011). Andromeda: A peptide search engine integrated into the MaxQuant environment. J. Proteome Res. Faull, S. V., Lau, A.M.C., Martens, C., Ahdash, Z., Hansen, K., Yebenes, H., Schmidt, C., Beuron, F., Cronin, N.B., Morris, E.P., et al. (2019). Structural basis of Cullin 2 RING E3 ligase regulation by the COP9 signalosome. Nat. Commun. Formstecher, E., Aresta, S., Collura, V., Hamburger, A., Meil, A., Trehin, A., Reverdy, C., Betin, V., Maire, S., Brun, C., et al. (2005). Protein interaction mapping: A Drosophila case study. Genome Res. genome through exhaustive two- hybrid screens. Nat. Genet.

980 Gazda, L., Pokrzywa, W., Hellerschmied, D., Löwe, T., Forné, I., Mueller-Planitz, F., Hoppe, T., and Clausen, T. (2013). The myosin chaperone UNC-45 is organized in tandem modules to support myofilament formation in C. elegans. Cell.

982 Goetz, A.W., Williamson, M.J., Xu, D., Poole, D., Grand, S.L., and Walker, R.C. (2012). Routine microsecond molecular dynamics simulations with AMBER - part 1: Generalized Born. J. Chem. Theory Comput. Graf, C., Stankiewicz, M., Nikolay, R., and Mayer, M.P. (2010). Insights into the conformational dynamics of the E3 ubiquitin ligase CHIP in complex with chaperones and E2 enzymes. Biochemistry. ubiquitin ligase Ufd2 interacts with the ubiquitin-like domains of Rad23 and Dsk2 via a novel and distinct ubiquitin-like binding domain. J. Biol. Chem. Hatakeyama, S., Yada, M., Matsumoto, M., Ishida, N., and Nakayama, K.I. (2001). U Box Proteins as a New Family of Ubiquitin-Protein Ligases. J. Biol. Chem.

994 Hellerschmied, D., Roessler, M., Lehner, A., Gazda, L., Stejskal, K., Imre, R., Mechtler, K., Dammermann, A., and Clausen, T. (2018). UFD-2 is an adaptor-assisted E3 ligase targeting unfolded proteins. Nat. Commun.

Hoppe, T., Cassata, G., Barral, J.M., Springer, W., Hutagalung, A.H., Epstein, H.F., and multiubiquitylation complex in C. elegans. Cell. Houde, D., Berkowitz, S.A., and Engen, J.R. (2011). The utility of hydrogen/deuterium exchange mass spectrometry in biopharmaceutical comparability studies. J. Pharm. Sci. Huerta-Cepas, J., Szklarczyk, D., Heller, D., Hernández-Plaza, A., Forslund, S.K., Cook, H., 
1005 Hughes, C.S., Foehr, S., Garfield, D.A., Furlong, E.E., Steinmetz, L.M., and Krijgsveld, J. 1006 (2014). Ultrasensitive proteome analysis using paramagnetic bead technology. Mol. Syst. Biol.

1007 Jiang, J., Ballinger, C.A., Wu, Y., Dai, Q., Cyr, D.M., Höhfeld, J., and Patterson, C. (2001).

1008 CHIP is a U-box-dependent E3 ubiquitin ligase: Identification of Hsc70 as a target for

1009 ubiquitylation. J. Biol. Chem.

1010 Joshi, V., Amanullah, A., Upadhyay, A., Mishra, R., Kumar, A., and Mishra, A. (2016). A decade

1011 of boon or burden: What has the chip ever done for cellular protein quality control mechanism

1012 implicated in neurodegeneration and aging? Front. Mol. Neurosci.

1013 Kamadurai, H.B., Qiu, Y., Deng, A., Harrison, J.S., MacDonald, C., Actis, M., Rodrigues, P.,

1014

1015 Miller, D.J., Souphron, J., Lewis, S.M., et al. (2013). Mechanism of ubiquitin ligation and lysine prioritization by a HECT E3. Elife.

1018 Kamath, R.S., and Ahringer, J. (2003). Genome-wide RNAi screening in Caenorhabditis elegans. Methods.

Kerscher, O., Felberbaum, R., and Hochstrasser, M. (2006). Modification of proteins by ubiquitin and ubiquitin-like proteins. Annu. Rev. Cell Dev. Biol. Koegl, M., Hoppe, T., Schlenker, S., Ulrich, H.D., Mayer, T.U., and Jentsch, S. (1999). A novel ubiquitination factor, E4, is involved in multiubiquitin chain assembly. Cell.

1024 Komander, D. (2009). The emerging complexity of protein ubiquitination. Biochem. Soc. Trans. Liao, Y., Wang, J., Jaehnig, E.J., Shi, Z., and Zhang, B. (2019). WebGestalt 2019: gene set analysis toolkit with revamped UIs and APIs. Nucleic Acids Res.

Liu, J., and Nussinov, R. (2011). Flexible cullins in cullin-RING E3 ligases allosterically regulate ubiquitination. J. Biol. Chem. Liu, C., Liu, W., Ye, Y., and Li, W. (2017). Ufd2p synthesizes branched ubiquitin chains to promote the degradation of substrates modified with atypical chains. Nat. Commun. Maier, J.A., Martinez, C., Kasavajhala, K., Wickstrom, L., Hauser, K.E., and Simmerling, C. (2015). ff14SB: Improving the Accuracy of Protein Side Chain and Backbone Parameters from ff99SB. J. Chem. Theory Comput.

1032 Murata, S., Minami, Y., Minami, M., Chiba, T., and Tanaka, K. (2001). CHIP is a chaperonedependent E3 ligase that ubiquitylates unfolded protein. EMBO Rep. Narayan, V., Landré, V., Ning, J., Hernychova, L., Muller, P., Verma, C., Walkinshaw, M.D., dependent E3-ubiquitin ligase activity of carboxy-terminus of Hsc70-interacting protein (CHIP). Mol. Cell. Proteomics.

Nikolay, R., Wiederkehr, T., Rist, W., Kramer, G., Mayer, M.P., and Bukau, B. (2004). Dimerization of the Human E3 Ligase CHIP via a Coiled-coil Domain Is Essential for Its Activity. J. Biol. Chem. Notredame, C., Higgins, D.G., and Heringa, J. (2000). T-coffee: A novel method for fast and accurate multiple sequence alignment. J. Mol. Biol. the conformation and oligomerization of e2 ubiquitin conjugates. Biochemistry. 
1052 Inuganti, A., Griss, J., Mayer, G., Eisenacher, M., et al. (2019). The PRIDE database and related tools and resources in 2019: Improving support for quantification data. Nucleic Acids Res. 47,

1054 D442-D450.

1055 Puchała, W., Burdukiewicz, M., Kistowski, M., Dąbrowska, K.A., Badaczewska-Dawid, A.E.,

1056 Cysewski, D., and Dadlez, M. (2020). HaDeX: An R package and web-server for analysis of data

1057 from hydrogen-deuterium exchange mass spectrometry experiments. Bioinformatics.

1058 Rain, J.C., Selig, L., De Reuse, H., Battaglia, V., Reverdy, C., Simon, S., Lenzen, G., Petel, F.,

1059 Wojcik, J., Schächter, V., et al. (2001). The protein-protein interaction map of Helicobacter

1060 pylori. Nature.

1061 Richly, H., Rape, M., Braun, S., Rumpf, S., Hoege, C., and Jentsch, S. (2005). A series of

1062

1063

ubiquitin binding factors connects CDC48/p97 to substrate multiubiquitylation and proteasomal targeting. Cell.

1064

1065

1066

1067

Ryckaert, J.P., Ciccotti, G., and Berendsen, H.J.C. (1977). Numerical integration of the cartesian equations of motion of a system with constraints: molecular dynamics of n-alkanes. J. Comput. Phys.

1068

Salomon-Ferrer, R., Götz, A.W., Poole, D., Le Grand, S., and Walker, R.C. (2013). Routine

1069 microsecond molecular dynamics simulations with AMBER on GPUs. 2. Explicit solvent particle mesh ewald. J. Chem. Theory Comput.

1070

1071 Scheufler, C., Brinker, A., Bourenkov, G., Pegoraro, S., Moroder, L., Bartunik, H., Hartl, F.U., and Moarefi, I. (2000). Structure of TPR domain-peptide complexes: Critical elements in the assembly of the Hsp70-Hsp90 multichaperone machine. Cell. Soss, S.E., Yue, Y., Dhe-Paganon, S., and Chazin, W.J. (2011). E2 conjugating enzyme selectivity and requirements for function of the E3 ubiquitin ligase CHIP. J. Biol. Chem. Tawo, R., Pokrzywa, W., Kevei, É., Akyuz, M.E., Balaji, V., Adrian, S., Höhfeld, J., and Hoppe, T. (2017). The Ubiquitin Ligase CHIP Integrates Proteostasis and Aging by Regulation of Insulin Receptor Turnover. Cell. Tehlivets, O. (2011). Homocysteine as a Risk Factor for Atherosclerosis: Is Its Conversion to S Adenosyl- L -Homocysteine the Key to Deregulated Lipid Metabolism? . J. Lipids. Di Tommaso, P., Moretti, S., Xenarios, I., Orobitg, M., Montanyola, A., Chang, J.M., Taly, J.F., and Notredame, C. (2011). T-Coffee: A web server for the multiple sequence alignment of protein and RNA sequences using structural information and homology extension. Nucleic Acids Res.

Visram, M., Radulovic, M., Steiner, S., Malanovic, N., Eichmann, T.O., Wolinski, H., Rechberger, G.N., and Tehlivets, O. (2018). Homocysteine regulates fatty acid and lipid metabolism in yeast. J. Biol. Chem. Vojtek, A.B., and Hollenberg, S.M. (1995). Ras-Raf Interaction: Two-Hybrid Analysis. Methods Enzymol. Vrablik, T.L., Petyuk, V.A., Larson, E.M., Smith, R.D., and Watts, J.L. (2015). Lipidomic and proteomic analysis of Caenorhabditis elegans lipid droplets and identification of ACS-4 as a lipid droplet-associated protein. Biochim. Biophys. Acta - Mol. Cell Biol. Lipids. Waterhouse, A., Bertoni, M., Bienert, S., Studer, G., Tauriello, G., Gumienny, R., Heer, F.T., De Beer, T.A.P., Rempfer, C., Bordoli, L., et al. (2018). SWISS-MODEL: Homology modelling of protein structures and complexes. Nucleic Acids Res.

Waterhouse, A.M., Procter, J.B., Martin, D.M.A., Clamp, M., and Barton, G.J. (2009). Jalview Version 2-A multiple sequence alignment editor and analysis workbench. Bioinformatics.

1098 Wojcik, J., Boneca, I.G., and Legrain, P. (2002). Prediction, assessment and validation of protein interaction maps in bacteria. J. Mol. Biol. 
1099 Xu, Z., Devlin, K.I., Ford, M.G., Nix, J.C., Qin, J., and Misra, S. (2006). Structure and 1100 Interactions of the helical and U-box domains of CHIP, the C terminus of HSP70 interacting 1101 protein. Biochemistry.

1102 Ye, Z., Needham, P.G., Estabrooks, S.K., Whitaker, S.K., Garcia, B.L., Misra, S., Brodsky, J.L., 1103 and Camacho, C.J. (2017). Symmetry breaking during homodimeric assembly activates an E3 1104 ubiquitin ligase. Sci. Rep.

1105 Zhang, H., Amick, J., Chakravarti, R., Santarriaga, S., Schlanger, S., McGlone, C., Dare, M., 1106 Nix, J.C., Scaglione, K.M., Stuehr, D.J., et al. (2015). A Bipartite Interaction between Hsp70 and 1107 CHIP Regulates Ubiquitination of Chaperoned Client Proteins. Structure.

1108 Zhang, H., Liu, Z., Ma, S., Zhang, H., Kong, F., He, Y., Yang, X., Wang, Y., Xu, H., Yang, A., et 1109 al. (2016). Ratio of S-adenosylmethionine to S-adenosylhomocysteine as a sensitive indicator of 1110 atherosclerosis. Mol. Med. Rep.

1111 Zhang, M., Windheim, M., Roe, S.M., Peggie, M., Cohen, P., Prodromou, C., and Pearl, L.H. 1112 (2005). Chaperoned ubiquitylation - Crystal structures of the CHIP U box E3 ubiquitin ligase and 1113 a CHIP-Ubc13-Uev1a complex. Mol. Cell. 
A

\begin{tabular}{|c|c|c|c|}
\hline & $\mathrm{CHN}-1$ & & CHN-1+UFD-2 \\
\hline JBE2L & & & \\
\hline & $-\quad-\quad+$ & & $-\quad+$ \\
\hline
\end{tabular}

B
UBE2D
Reaction (mins):
$\begin{array}{cccccccccccc}+ & + & + & + & + & + & + & + & + & + & + & + \\ 60 & 90 & 180 & 60 & 90 & 180 & 60 & 90 & 180 & 60 & 90 & 180\end{array}$

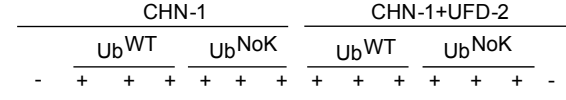

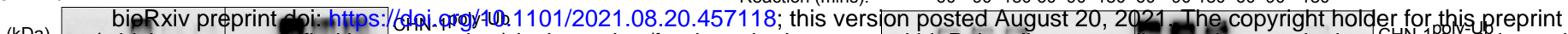
(kDa) (which was not ceftified by peer review) is the author/funder, who has granted bioRxiv a license to display the preprint in perpedetuity. available under aCC-BY-NE94-0 International license.

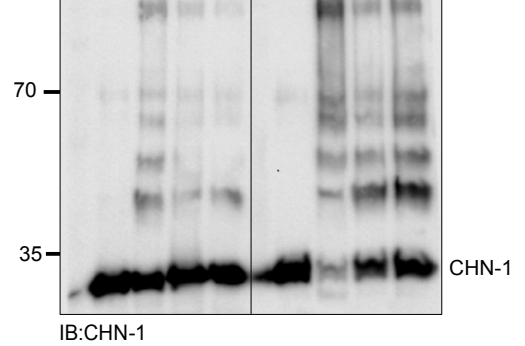

C

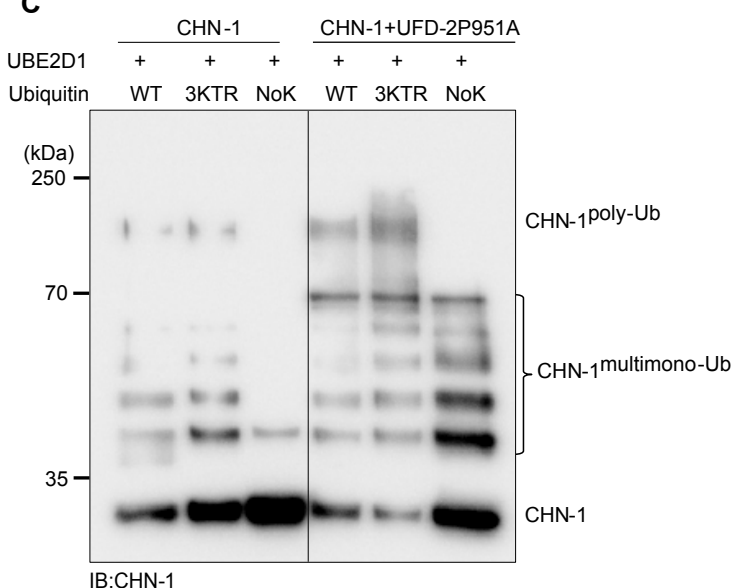

D

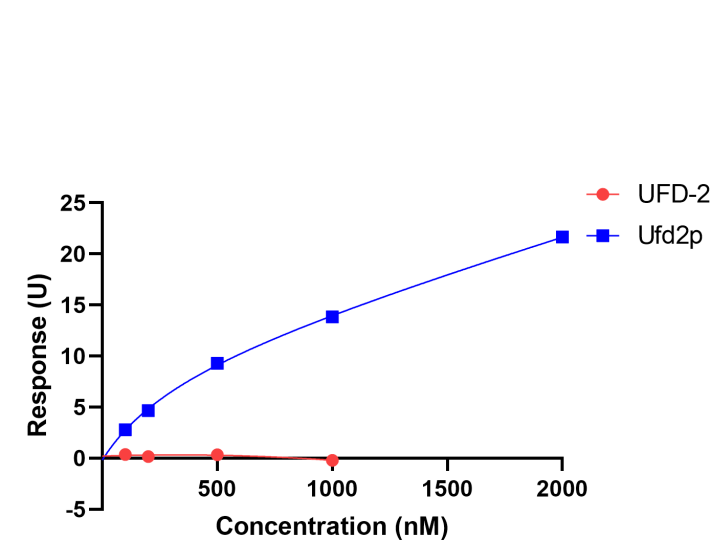

E

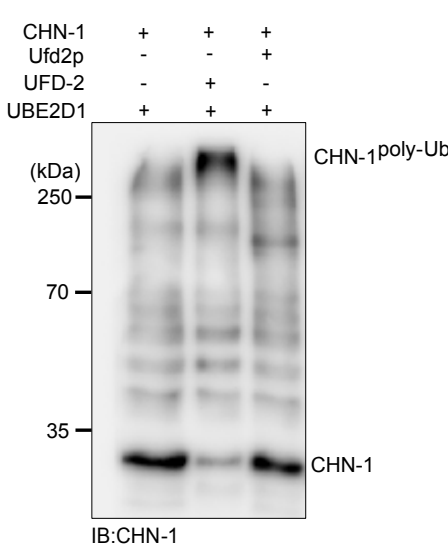




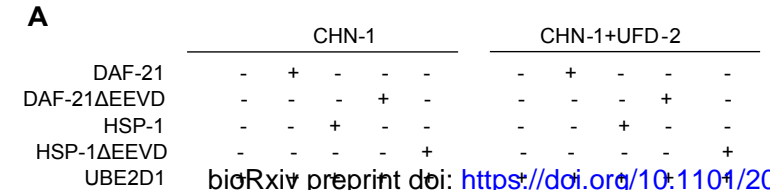

bioRxiv preprint doi: httpst//dot.org/10+110 /2021.08.20.457118; this versianplosted August 20, 2021. The copyright holder for this preprint
(which was not certified by peer review) is the author/funder who has granted bioRxiv a license to display the preprint in perpetuity. It is made

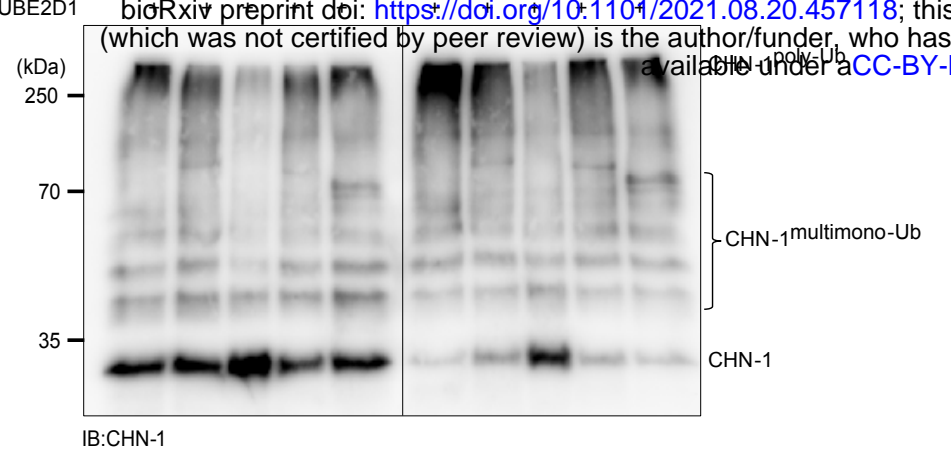

B aa aa
640
HSP-1 PEPTIDE - - + + $\frac{\text { AGGAGGPTIEEVD }}{\text { HSP-1 PEPTIDE }}$

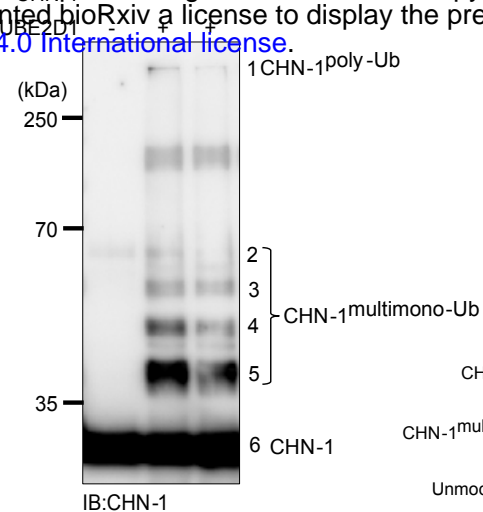

C UFD-2 ${ }^{86}$

aa FHNAIECMRKNSIVGFSQLESFEHLAEDVKKEYEAKAELEEEYDDVPEEF $\frac{\text { SQLESFEHLAEDV }}{\text { PEPTIDE1 }} \frac{\text { KKEYEAKAELEEEYDDVP }}{\text { PEPTIDE2 }}$

UFD-2 ${ }^{\text {aa }}{ }_{\text {NLLMCMI KFYSDFEDNG DFYEKFNVRGNIQ }}^{\text {aa }}$ $\frac{\text { KFYSDFEDNG }}{\text { PEPTIDE3 }}$
D

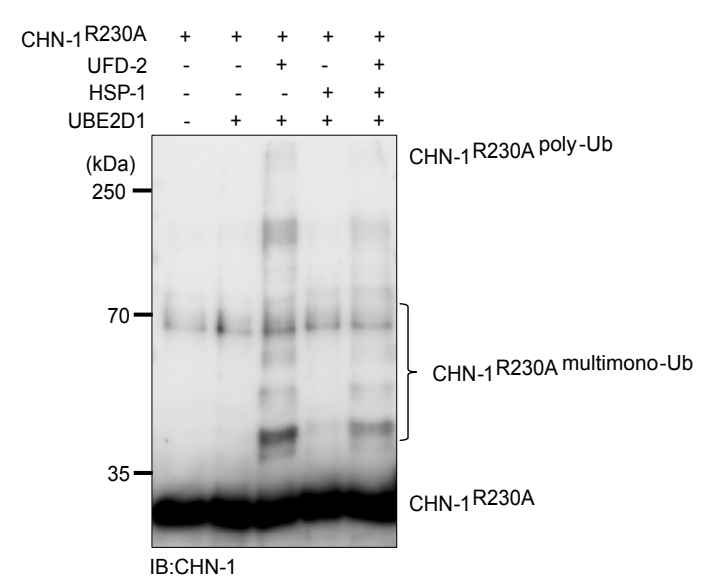

E

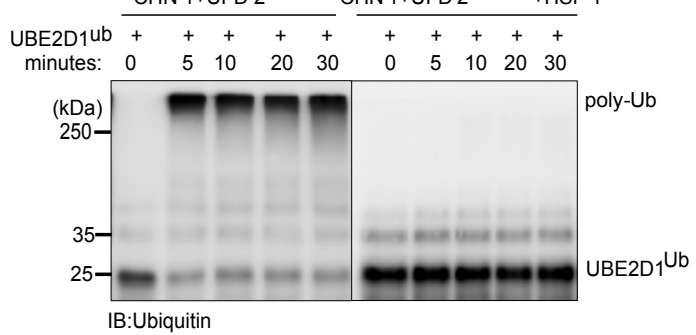

aa $\quad P$
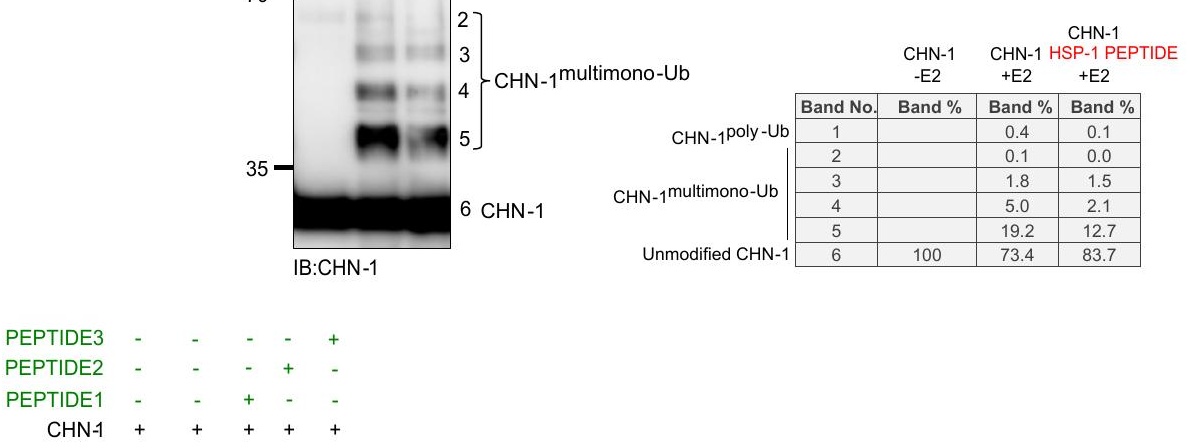

UBE2D

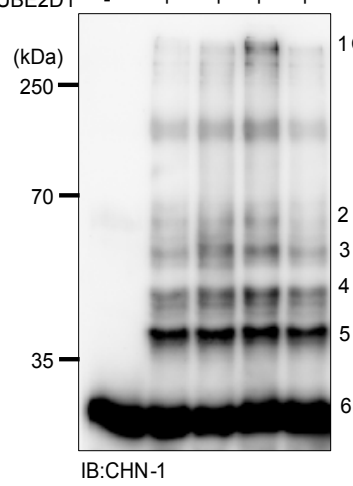

$1 \mathrm{CHN}-1$ poly $-\mathrm{Ub}$

$\mathrm{CHN}-1$ multimono-Ub

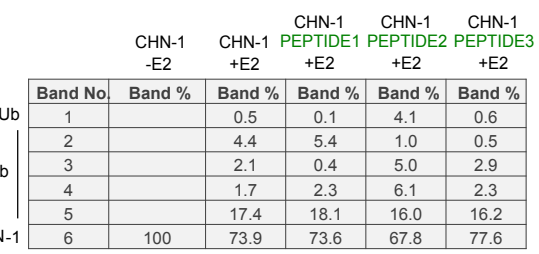

$\mathbf{F}$

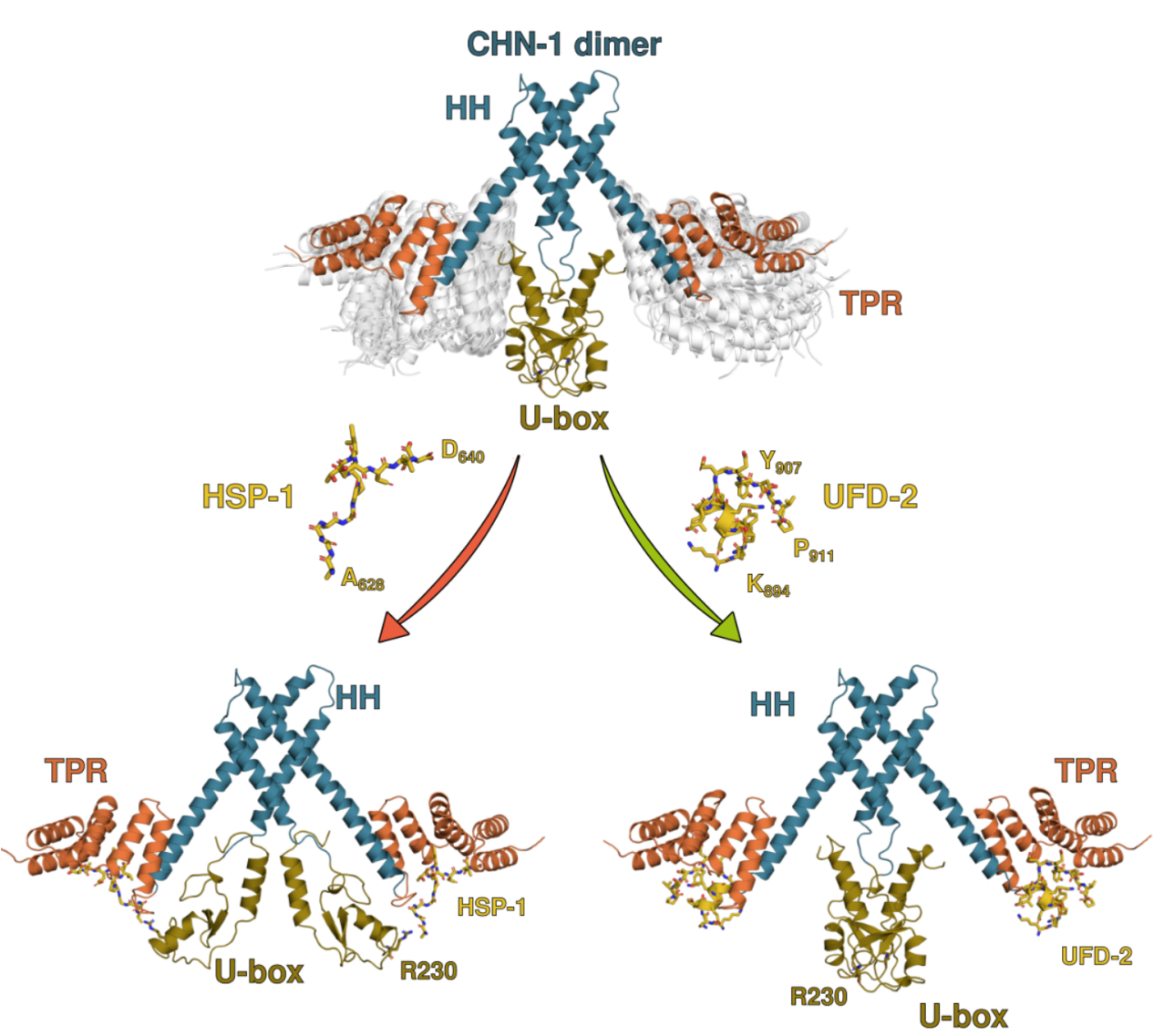

Steady-state closed conformation/low activity 


\section{A}

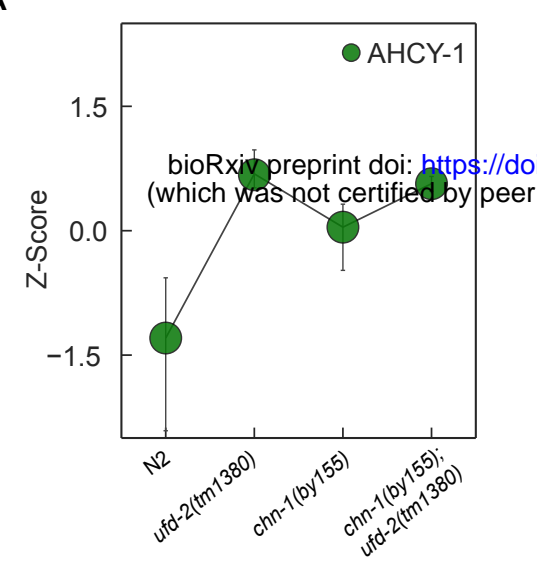

C
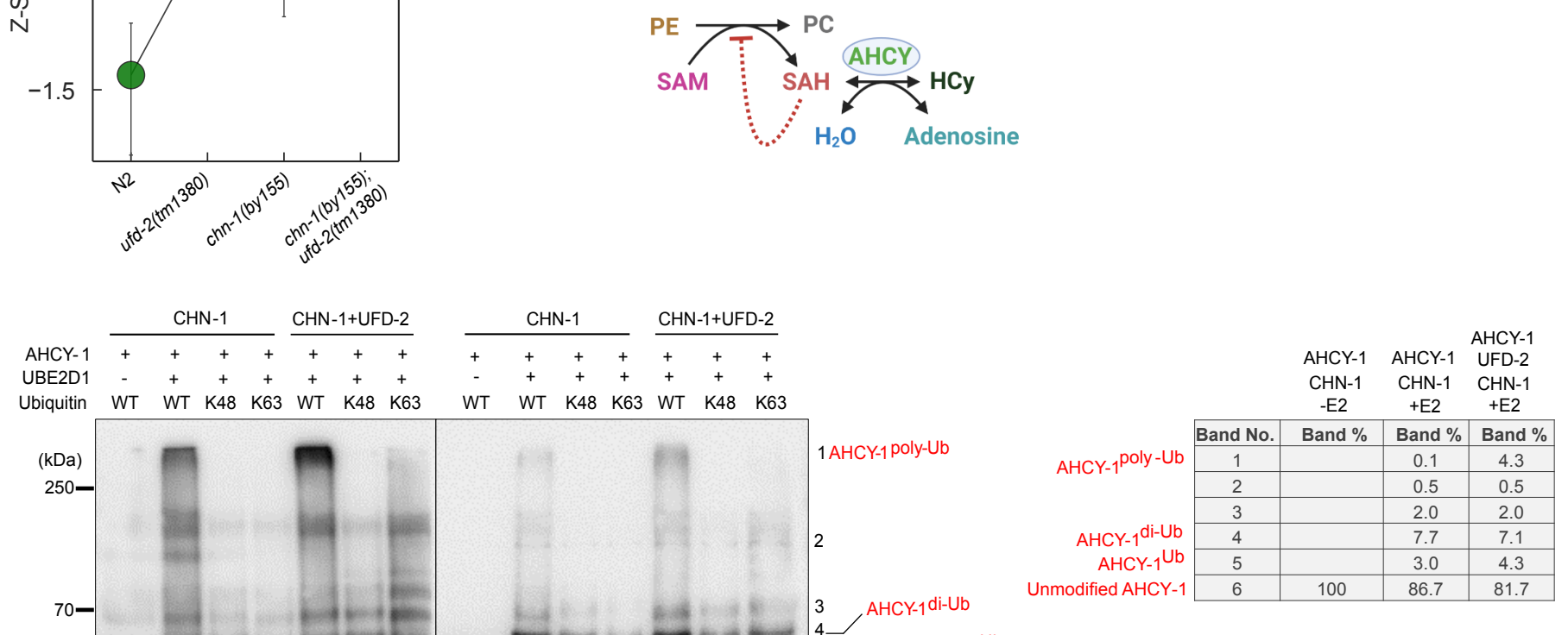

D
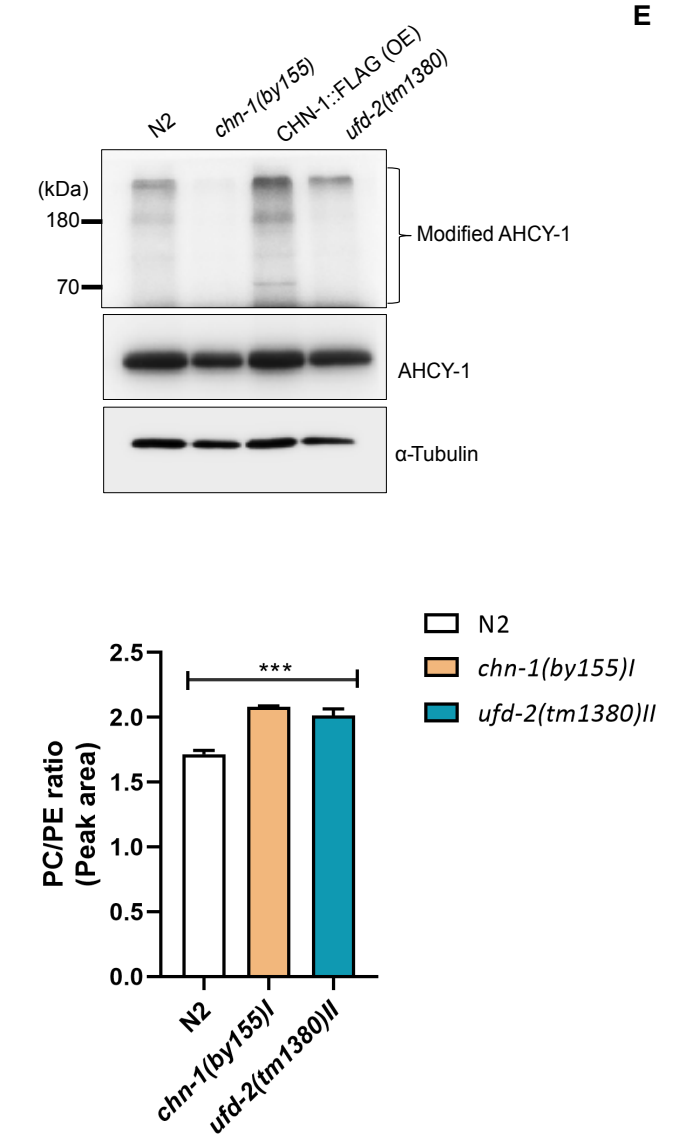

E

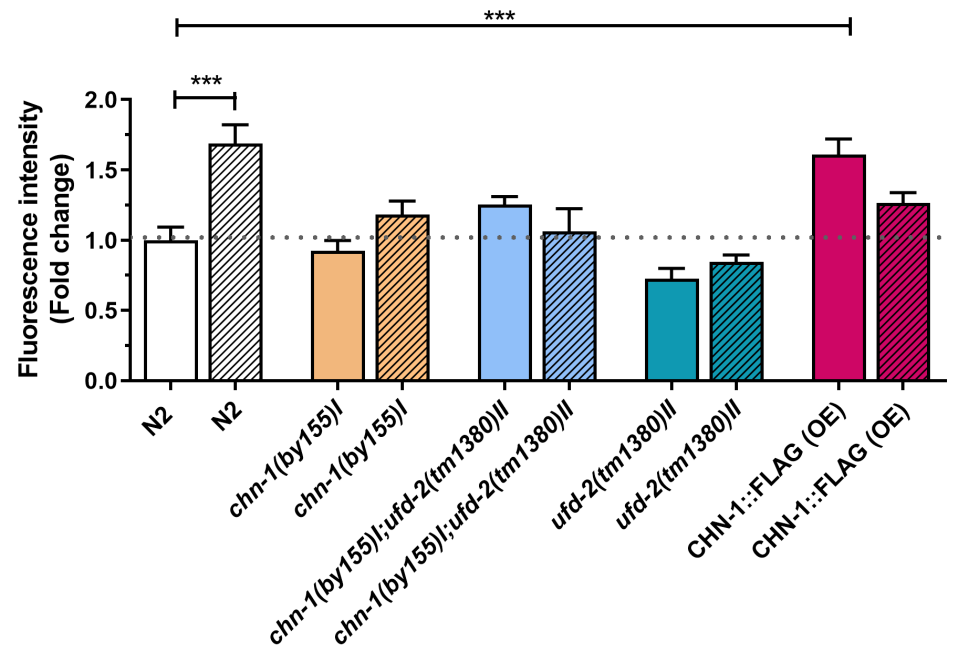

$\square$ Control RNAi ahcy-1 RNAi 